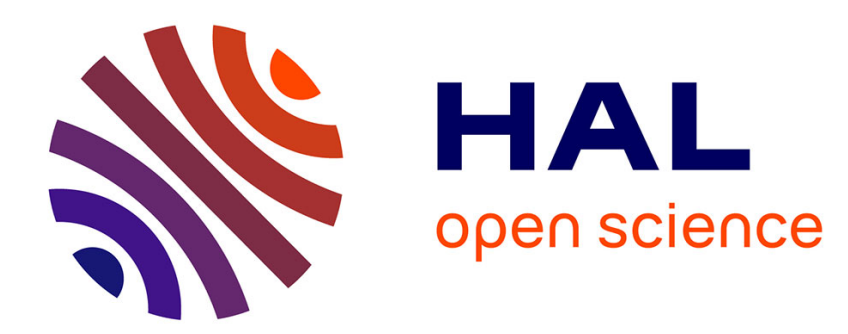

\title{
Un état des comptes du royaume de Chypre en 1412-1413 \\ Gilles Grivaud
}

\section{To cite this version:}

Gilles Grivaud. Un état des comptes du royaume de Chypre en 1412-1413. Bulletin de Correspondance Hellenique, 1998, 122 (1), pp.377 - 401. 10.3406/bch.1998.7178 . hal-01937225

\section{HAL Id: hal-01937225}

\section{https://hal-normandie-univ.archives-ouvertes.fr/hal-01937225}

Submitted on 28 Nov 2018

HAL is a multi-disciplinary open access archive for the deposit and dissemination of scientific research documents, whether they are published or not. The documents may come from teaching and research institutions in France or abroad, or from public or private research centers.
L'archive ouverte pluridisciplinaire HAL, est destinée au dépôt et à la diffusion de documents scientifiques de niveau recherche, publiés ou non, émanant des établissements d'enseignement et de recherche français ou étrangers, des laboratoires publics ou privés. 


\section{Un état des comptes du royaume de Chypre en 1412-1413}

In: Bulletin de correspondance hellénique. Volume 122, livraison 1, 1998. pp. 377-401.

Citer ce document / Cite this document :

Grivaud Gilles. Un état des comptes du royaume de Chypre en 1412-1413. In: Bulletin de correspondance hellénique. Volume 122, livraison 1, 1998. pp. 377-401.

doi : $10.3406 /$ bch.1998.7178

http://www.persee.fr/web/revues/home/prescript/article/bch_0007-4217_1998_num_122_1_7178 


\section{Abstract}

A Venetian copy of the balance-sheet drawn up by the Comptroller of the kingdom of Cyprus for the year 1412-1413 shows the ability of the these last Lusignans to react to the crises of the 14 th $\mathrm{c}$. The statement, in spite of numerous lacunae, makes it clear that Janus, following the example of Jaques I, intensified the direct exploitation of the crowns domain, taxed the serfs and introduced extraordinary levies (royal tithe, salt tax). Thus, during the second decade of the 15th $\mathrm{c}$, the Frankish kingdom received considerable revenues comparable to those of other States in the medieval West. Janus's reign, at least until 1424, is not therefore to be identified with the period of decline so often described.

\section{Résumé}

Une copie vénitienne d'un bilan financier, dressé par la Secrète du royaume de Chypre pour l'année 1412-1413, vient démontrer les capacités de réaction des derniers Lusignan aux crises du XIVe s. Le compte examiné, en dépit de ses nombreuses lacunes, assure que Janus, suivant l'exemple de Jacques ler, renforce l'exploitation directe du domaine de la couronne, fiscalise les fiefs, développe les recettes extraordinaires (dîme royale, mète du sel). Ainsi, durant la seconde décennie du XVe s., le royaume franc conserve des revenus importants, comparables à ceux d'autres États de l'Occident médiéval. Le règne de Janus, au moins jusqu'en 1424, ne s'identifie pas à la période de déclin trop souvent décrite.

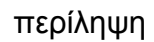

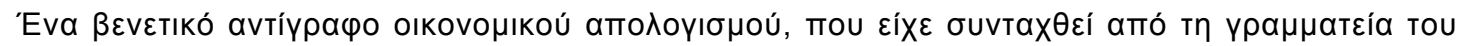

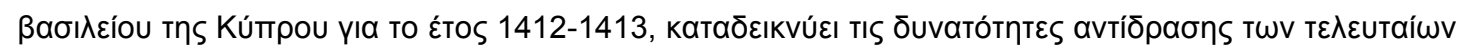

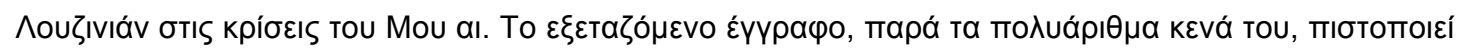

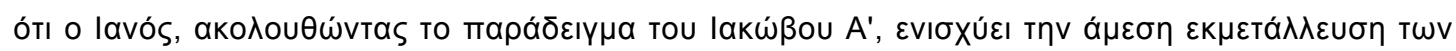

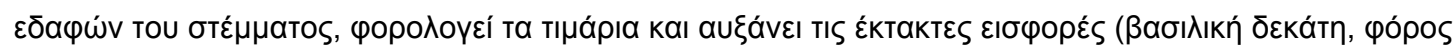

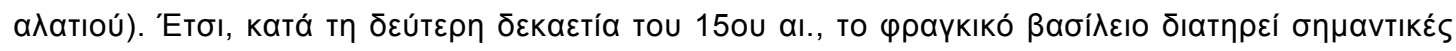

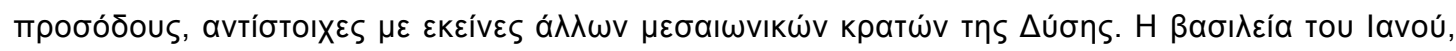

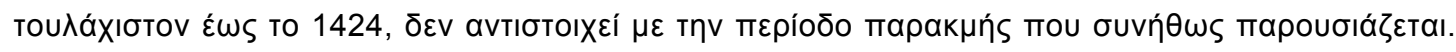

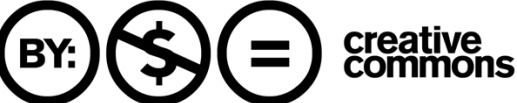




\section{Un état des comptes du royaume de Chypre en 1412-1413*}

par Gilles GRIVAUD

Les historiens s'accordent pour distinguer deux phases majeures dans la vie économique du royaume de Chypre: un essor régulier, engagé dès le début du XIII ${ }^{\mathrm{e}}$. et qui culmine sous les règnes de Henri II (1285-1324) et Hugues IV (1324-1359), puis une période de déclin, qui couvre la seconde moitié du XIV s. et tout le XVe. Cette dépression résulte de l'association de plusieurs facteurs: une violente chute démographique causée par la récurrence des épidémies de peste, le détournement des trafics maritimes vers l'Égypte et la Syrie, les guerres contre Gênes (1373-1374, 1403) et les Mamelouks (1424-1426). Cette série de catastrophes place peu à peu les Lusignan sous la dépendance des banquiers vénitiens, facilitant la mainmise de la Sérénissime sur l'île (1474)1.

* Abréviations utilisées:

AMADI = Chroniques d'Amadi et de Strambaldi (éd. R. DE MAS-LATRIE), I (1891).

ASV = Archivio di Stato di Venezia.

F. BUSTRON = F. BuSTRON, Chronique de l'île de Chypre (éd. R. DE MAS-LATRIE) (1886).

Correr = Civico Museo Correr (Venise).

L. DE MAS-LATRIE, Histoire = L. DE MAS-LATRIE, Histoire de l'île de Chypre sous le règne des princes de la maison de Lusignan, I-III, (1852-1861).

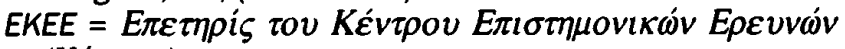
(Kútoov).

G. GRIVAuD, "Ordine della Secreta di Cipro" = G. GRIVAUD, "Ordine della Secreta di Cipro. Florio Bustron et les institutions franco-byzantines afférentes au régime agraire de

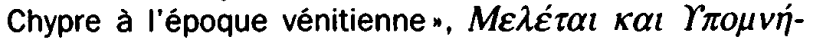

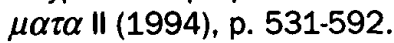

G. HILL = G. HILL, A History of Cyprus, II-III (1952).

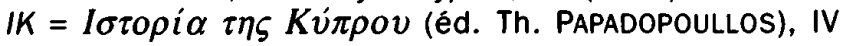
(1995), V (1996).

MACHAIRAS $=$ L. MACHAIRAS, Recital concerning the Sweet Land of Cyprus entitled Kronaka (éd. R. M. DAWKINS), |-II (1932).

Marciana = Biblioteca Nazionale Marciana (Venise).

RHCLois = Recueil des historiens des croisades, Lois (éd. comte A. BEUGNOT), HII (1841-1843).

J. RICHARD, Documents chypriotes = J. RICHARD, Documents chypriotes des archives du Vatican XIV et $X V^{e}$ siècles (1962).

J. RICHARD, Le Livre des Remembrances = J. RICHARD, Le Livre des Remembrances de la Secrète du royaume de Chypre (1468-1469) (1983).

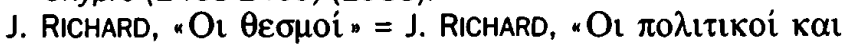

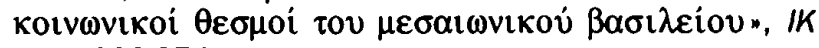
IV, p. 333-374.

STRAMBALDI = Chroniques d'Amadi et de Strambaldi (éd. R. DE MAS-LATRIE), II (1893).

Le document à l'origine de la présente publication avait déjà été repéré, il y a une dizaine d'années, par Benjamin Arbel; nous lui adressons nos plus vifs remerciements pour nous avoir laissé éditer ce compte que lui-même se proposait de publier. Nos remerciements aussi à Michel Balard, Roland Étienne, Geneviève Lang, Chryssa A. Maltézou, Marie-Rose Papadakis et Jean Richard pour leurs remarques, à Elissavet Chryssikopolou pour son aide.

1 L. DE MAS-LATRIE, Histoire I, p. 390-391, 511-512; W. HEYD, Histoire du commerce du Levant au Moyen Âge (tr. fr. de FURCY-RAYNAUD) (1885-1886), I, p. 359-365, II, p. 323, 407-426; G. HILL, II, p. 194-195, 207, 292, 369; D. JENNESS, The economy of Cyprus. A survey to 1914 (1962), p. 23-42; J.-C. HOCQUET, Le sel et la fortune de 
Dans ses grandes lignes, ce schéma s'appuie sur l'observation du commerce du Levant tel qu'il peut être reconstitué à partir des archives italiennes; les récits des chroniqueurs chypriotes, voire des voyageurs, ont apporté des informations complémentaires mais il faut bien reconnaitre que, dans leur ensemble, les sources utilisées proviennent de milieux étrangers aux institutions administratives du royaume franc. Comme le remarquait récemment David Jacoby, la connaissance de l'économie chypriote médiévale reste largement tributaire de notre perception des échanges commerciaux ${ }^{2}$. La découverte d'un état des comptes du royaume pour l'année fiscale 1412 vient donc fournir un précieux élément de compréhension des finances des Lusignan, lorsque l'île entre dans une phase de déclin économique et politique que le règne de Janus accentue après 1426.

À plus d'un titre, Janus peut être considéré comme un prince marquant de la famille des Lusignan. Né à Gênes vers 1374-1375, durant la captivité de Jacques ${ }^{\text {er }}$ et d'Helvis de Brunswick, il passe sa jeunesse en exil ; les clauses du traité de 1381 l'avaient transformé en otage, expérience cruelle qui lui permit cependant de recevoir une éducation à l'italienne; Janus deviendra un monarque raffiné, sans doute le plus distingué de la dynastie. Il gagne Chypre en 1392, épouse Anglesia Visconti, sœur de la duchesse de Milan, en 1400, puis Charlotte de Bourbon, filleule de Charles VI, le 25 août 1411. Jusqu'au décès de la reine, en 1422, le couple entretient une vie de cour brillante, conjuguant les charmes émollients du luxe d'Orient aux modes lancées par les princes français et italiens du Moyen Âge finissant. Cette période heureuse s'achève vite; le 6 juillet 1426, Janus sort vaincu de la bataille de Choirokitia et subit l'humiliation des geôles cairotes; le roi doit accepter la suzeraineté du sultan et lui verser tribut. 1426 marque une nouvelle période de crise pour Chypre: crise politique, puisque le royaume franc perd son indépendance; crise sociale, car la défaite de Choirokitia déclenche une révolte paysanne qui secoue l'île pendant dix mois; crise financière, enfin, puisque l'aristocratie doit verser d'énormes rançons pour libérer les nobles captifs au Caire; Janus, lui-même, est contraint de liquider ses joyaux à Gênes avant de lever de nouvelles aides. Les chroniqueurs affirment que de 1426 à sa mort, le 29 juin 1432, Janus ne sourit plus jamais ${ }^{3}$.

Janus accède au trône le 11 ou le 19 novembre 1398; il hérite d'un royaume dont la prospérité appartient au passé. Le conflit avec Gênes a plongé Chypre dans une crise financière sans précédent dont il convient de rappeler les effets. Par le traité du 21 octobre 1374, Famagouste,

Venise (1978), I, p. 196; B. ARBEL, "Traffici marittimi e sviluppo urbano a Cipro (secoli XIII-XVI) ", in E. POLEGGI (éd.), Città portuali del Mediterraneo, Storia e Archeologia, Atti del Convegno Internazionale di Genova 1985 (1989), p. 89-94; M. BALARD, "La place de Famagouste génoise dans le royaume des Lusignan, 1374-1464 ", in C. MUTAFIAN (éd.), Les Lusignan et l'Outre Mer (1993), p. 16-27; P. W. EDBURY,

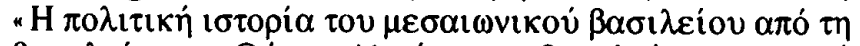

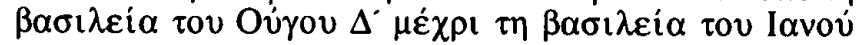
(1324-1432)", IK IV, p. 88-92.

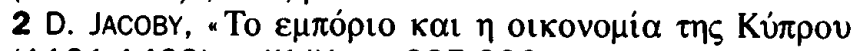
(1191-1489) ", IK IV, p. 387-388, et les judicieuses remarques sur la distinction qu'il convient d'opérer entre déclin commercial de Famagouste et essor de la production à Chypre aux p. 445-446.

3 Sur Janus et son règne: MACHAIRAS, § 628-702; AMADI, p. 496-515; STRAMBALDI, p. 262-287; F. BUSTRON, p. 354 371 ; E.-G. REY, Les familles d'Outremer de Du Cange (1849), p. 88-92; G. HILL, II, p. 447-496; W. H. RUDT DE COLLENBERG, "Les Lusignan de Chypre ", EKEE X (1979-80),

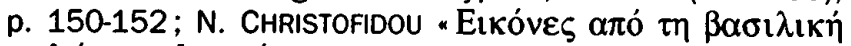

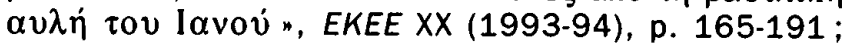
P. W. EDBURY, art. cit. (supra, n. 1), p. 145-154. La mention sur la vente des joyaux de Janus figure chez L. BALLETTO, "Les Génois dans l'île de Chypre au bas Moyen Âge ", in C. Mutafian (éd.), op. cit. (supra, n. 1), p. 33. 
son port et ses douanes passent sous contrôle génois et plus de 8 millions de besants sont exigés au titre des indemnités de guerre; pour trouver les fonds nécessaires, Pierre II (1361-1382) et Jacques $\mathrm{I}^{\text {er }}$ (1385-1398) recourent massivement à l'extraordinaire sans parvenir à éteindre une dette réduite à 4 millions de besants en 1383. En 1403, Janus tente de renverser le rapport de force avec Gênes en attaquant Famagouste mais l'échec se solde par de nouvelles exigences financières: le traité de 1403 impose au roi de verser plus de 3,8 millions de besants en dix annuités; le siège du port, jusqu'en 1406, n'ébranle pas l'intransigeance des Génois 4 .

À la lecture des chroniques, l'année 1412 appartient à une période calme de l'histoire du royaume. Les campagnes se rétablissent d'un retour de la peste et des ravages causés par des nuées de sauterelles qui avaient détruit les récoltes trois ans de suite, entre 1409 et 1411 . Léontios Machairas attribuait le retour des conditions favorables à Charlotte de Bourbon, dont l'arrivée à Chypre coïncidait avec le reflux des acridiens; par-delà l'influence bienfaisante de la jeune reine, les mesures décrétées par Janus pour collecter œufs et larves expliquent ce succès 5 . En matière diplomatique, les relations avec Gênes s'étaient normalisées; Janus avait compris qu'il ne pourrait se dégager des Génois et effectuait des versements aux massari de Famagouste, à dates fort irrégulières cependant ${ }^{6}$. Depuis 1404, il manifestait ses humeurs belliqueuses par de fréquentes expéditions contre le littoral mamelouk, soutenant aussi les opérations des corsaires catalans. De prime abord, l'état des comptes de l'année 1412 reflète la situation financière d'un royaume en phase de redressement; le récit laissé par le chancelier Luchino dal Campo sur la visite à Nicosie du marquis Nicolò III d'Este illustre l'éclat que la cour chypriote conservait aux yeux du prince ferrarais ${ }^{7}$. Tous les chroniqueurs rejoignent Florio Bustron pour assimiler les onze années de mariage de Janus et Charlotte à une période heureuse pour l'ensemble du royaume ${ }^{8}$.

\section{Le document et son contexte}

Le texte qui nous retient ici appartient à un volume de miscellanea sur le Regno di Cipro, déposé aux archives du musée Correr, sous la cote Cicogna 3596. Le manuscrit rassemble 30 pièces qui traitent différents aspects de l'histoire de Chypre au XVI s. ; pêle-mêle, s'y côtoient des avis relatifs à la défense de l'île, des résumés de la chronique de Florio Bustron, des copies de rapports très diffusés à l'époque (tels ceux de Francesco Attar, Antonio Zane, Ascanio Savorgnan,

4 La littérature historique sur les différents traités passés entre Gênes et les Lusignan est abondante; nous renvoyons à l'étude récente de $C$. OTTEN-FrouX qui fait un point définitif sur la question: "Les relations politico-financières de Gênes avec le Royaume des Lusignan (1374-1460) m, in M. BALARD, A. DUCELLIER (éds), Coloniser au Moyen Âge (1995), p. 61-75. 5 MACHAIRAS, $\$ 637-639$.

6 C. OTTEN-Froux, art. cit. (supra, n. 4), p. 71-72, relève, dans les registres des massari de Famagouste, les versements suivants pour la période 1403-1412 (en besants):
$1404: 49271 / 5$ b. ; 1405-1406: $55810 / 6$ b. ; 1406 : $53653 / 15$ b. ; mars - juillet $1411: 30333 / 8$ b.

7 La visite à Chypre de Nicolò d'Este se déroule du 26 mai au 3 juin 1412: G. GRIVAUD, Excerpta Cypria Nova, Voyageurs occidentaux à Chypre au xve siècle (1990), I, p. 41-49.

8 "In tempo della qual regina era pacifico el paese tutto, et era anco abbondantia d'ogni cose ": F. BUSTRON, p. 356 ; MACHAIRAS, § 638; AMADI, p. 498. 
Bernardo Sagredo), diverses pièces administratives. Ce matériel a été rassemblé au lendemain de la conquête de l'île par les Ottomans, car le recueil comprend les récits de la prise de Nicosie consignés par Giovanni Sozomeno (pièce $\mathrm{n}^{\circ} 21$ ) et Giovanni Falier (pièce $\mathrm{n}^{\circ} 22$ ), le célèbre discours tenu par Marc'Antonio Colonna pour justifier le détournement de la flotte chrétienne partie au secours de Famagouste, en date du 26 septembre 1570 (pièce n ${ }^{\circ} 5$ ).

La pièce qui porte le numéro 30 se présente sous la forme d'un petit cahier de 16 folios copiés de la même main (dimensions: 15,6 x 21,3 cm), intitulé Delle cose di Cipro; aux f. 1r-3r, se trouve l'état abrégé des comptes de l'année fiscale 1412 reproduit en appendice; il précède un relevé des recettes et des dépenses de la camera di Cipro pour l'année fiscale 1556 (f. 3v-5r). Suivent un état quinquennal des récoltes de blé et d'orge entre 1525 et 1565 (f. $5 \mathrm{v}$ ), un résumé des résultats du recensement de la population dressé en 1563 (f. Gr), une liste des fieffés et de leurs obligations de service (f. $7 \mathrm{r}-10 \mathrm{v}, 12 \mathrm{r}$ ), des notes éparses sur les productions insulaires (f. 11r-v). Cette collection de documents officiels, établie vers 1566 , reflète clairement les préoccupations de Venise après 1550, car le compte de 1412 fut copié pour fournir un pendant à celui de 1556; nul doute que l'examen comparé des deux bilans devait permettre à Venise de renforcer son contrôle sur les revenus de l'île, donc d'améliorer ses recettes fiscales; jusqu'en 1570, provéditeurs et syndics envoyés par la métropole déplorent la maîtrise insuffisante des vieux registres francs par les secrétaires vénitiens, situation qui facilite les usurpations orchestrées par les feudataires chypriotes aux dépens de la camera ${ }^{9}$.

Le nom du copiste du cahier $n^{\circ} 30$ n'est pas dévoilé par les documents, pas plus que l'identité du commanditaire ou les conditions d'établissement de la copie. Dans ces conditions, saisir l'origine du compte de 1412 relève de la conjecture; la prudence n'interdira pas quelques hypothèses pour mieux circonscrire l'environnement dans lequel le texte a été produit et transmis, distinguant ce qui appartient à la copie du Correr de ce qui relève de l'original chypriote.

Une première remarque permet d'affirmer que la copie du Correr a été dressée par un écrivain peu familier des systèmes de mesure en vigueur dans l'île; les confusions observées dans les résolutions des abréviations de poids et mesures (cantare/rotolus, mètre chypriote/salma sicilienne) excluent l'attribution de ce travail aux grands commis chypriotes de l'administration coloniale actifs à Nicosie, tels Florio Bustron ou Zuan Zamberlan ${ }^{10}$; l'ultime copie a été exécutée à Venise, vers 1566, par un secrétaire n'ayant sans doute jamais exercé à Chypre. À ce scribe peuvent être attribuées les observations sur l'absence de recettes tirées des salines et de Famagouste, répétées à deux reprises en fin de rubrique. Par ailleurs, l'accumulation de lectures erronées et d'interversions de chiffres suggère des versions intermédiaires du texte. De l'original français, une

9 ASV, Collegio V (Secreta), Relazioni, busta 84, fascicolo 541/776, rapport du luocotenente Marc'Antonio Trevisan, f. $1 \mathrm{v}$; L. DE MAS-LATRIE, Histoire, III, p. 551; V. LAMANSKY, Secrets d'État de Venise, documents, extraits, notices et études (1884), p. 622-630, 014-018; G. GRIVAUD, "Sur quelques contradictions de l'administration vénitienne à
Chypre (1473-1570) ", Thesaurismata 20 (1990), p. 195197.

10 Sur les services administratifs de Chypre à l'époque véni-

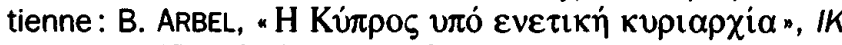
IV, p. 466-471; G. GRIVAUD, "Ordine della Secreta di Cipro", p. $541-546$. 
traduction italienne a été réalisée, sans doute à Nicosie où tous les registres de la Secrète étaient conservés; cette traduction peut appartenir aux années 1550-1560, quand Venise cherche à augmenter ses recettes ${ }^{11}$. Entre cette traduction et la copie vénitienne du Correr, on suppute une ou plusieurs copies qui répètent et multiplient les fautes.

Ces observations sur le texte du Correr ne suffisent pas à cerner l'environnement du compte de 1412 et il faut s'en remettre aux travaux de Jean Richard pour saisir comment a été produit le document. Les finances du royaume des Lusignan relèvent de la Secrète, bureau central chargé de l'administration du domaine et du trésor du roi; les tâches y sont réparties entre plusieurs collèges rassemblant une dizaine de secrétains ${ }^{12}$; parmi ceux-ci, certains doivent "tener li stadi cio è la entrada et uscita del Regno", pour reprendre les termes de Florio Bustron, en $1554^{13}$. Il s'agit donc d'un service comptable à compétence technique, exécutant les décisions arrêtées par les autres collèges de la Secrète; aucune charge d'enregistrement, autre que comptable, ne semble lui incomber puisque les mandats de paiement (apodixes) ou les reçus des paiements effectués au bénéfice du roi, au titre des assignations, sont établis par les autres collèges de secrétains et consignés dans les Livres des Remembrances, notamment dans Le Livre de paie et quitances $^{14}$. Les comptes des bailliages royaux, des fermes (apauts), des levées d'impôts - ordinaires ou non - lui échappent aussi ${ }^{15}$. En d'autres termes, ces secrétains se consacrent à noter les opérations d'entrée et de sortie du trésor; ils sont réunis en un collège réduit à un ou deux membres, peut-être placés sous la direction d'un trésorier; ainsi, en 1468, François de Tripoli est l'escrivain des yssues, Jean Strambailli le trezorier (il est déjà cité à cette charge en décembre 1449) ${ }^{16}$.

Faut-il attribuer au trésorier de la Secrète le document de 1412? Vraisemblablement, car sa fonction le désigne comme un officier suffisamment qualifié pour dresser ce type d'état mais il peut aussi avoir participé à sa composition sans l'avoir entièrement établi, laissant cette responsabilité au bailli ou au pourvéeur, chefs de la Secrète; pour chacun de ces officiers, le relevé permet, en effet, de rendre compte de son administration. Par ailleurs, si nous cherchons à définir le document du Correr, celui-ci correspond à un état d'exécution du trésor et non à un "budget "

11 Rappelons que, dans les années 1560, l'usage du français avait totalement disparu de Chypre; les lettrés capables de lire les vieux textes des Lusignan se limitent à deux ou trois personnes; ainsi, en 1565-1566, les Davila et les Podocataro recourent aux services d'un Savoyard de passage à Nicosie, Catherin Le Doux, pour obtenir la traduction de privilèges conservés dans leurs archives familiales,

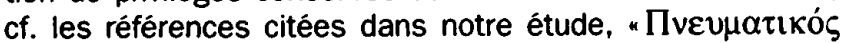

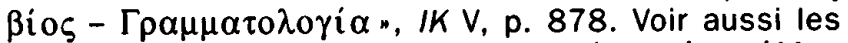
remarques de C. A. MALTEZOU, "'H $\pi \varepsilon \rho \imath \pi \dot{\varepsilon} \tau \varepsilon \imath \alpha \dot{\varepsilon} \operatorname{vo\zeta } \dot{\varepsilon} \lambda \lambda \eta$ -

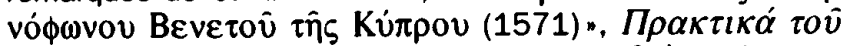

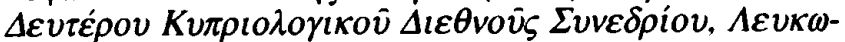

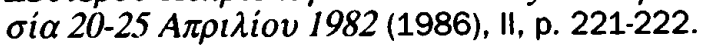

12 Sur les compétences de la Secrète: J. RICHARD, Le Livre des Remembrances, p. XI-XVII; G. GRIVAUD, "Ordine della Secreta di Cipro ", p. 561-565.
13 G. GRIVAud, "Ordine della Secreta di Cipro ", p. 561.28.

$14 \mathrm{~J}$. RICHARD, Le Livre des Remembrances, $\mathrm{p}$. IX-X et $n^{o s}$ 195-203; G. GRIVAUD, "Ordine della Secreta di Cipro", p. $563.40-43$.

15 Toujours selon Florio Bustron: G. GRIVAUD, "Ordine della Secreta di Cipron, p. 561.26-28, confirmé par la répartition des différentes charges en 1468: J. RICHARD, Le Livre des Remembrances, $\mathrm{n}^{\circ} 1$.

$16 \mathrm{~J}$. RICHARD, Le Livre des Remembrances, $\mathrm{n}^{\text {os }} 1,36,48$, 93,222 ; J. RICHARD, Documents chypriotes, p. 152. Nous hésitons cependant à reconnaître en Jaime Saplana un autre trésorier de la Secrète durant les années 1471-1472. lorsqu'il est cité "gubernatore regie camere": L. DE MASLATRIE, "Documents nouveaux servant de preuves à l'histoire de l'île de Chypren, in Mélanges historiques IV (1882), p. $410-411$. 
prévisionnel; les recettes se trouvent notées avec des valeurs précises, non avec les chiffres ronds qui caractérisent les estimations ${ }^{17}$. On peut avancer que le trésorier de la Secrète préparait un récapitulatif annuel des finances royales ou plus exactement, comme nous le verrons plus bas, de la partie des finances royales gérée par la Secrète car l'état de 1412 n'est que le corps central, principal, du "budget" du royaume. Dans ses grandes lignes, l'état des comptes chypriote peut être assimilé à l'extractus thesauri présenté chaque semestre aux rois de France, l'un et l'autre se définissant comme des documents comptables éclairant la situation du trésor vis-à-vis du roi ${ }^{18}$.

Compte tenu des exigences financières des Génois, les Lusignan avaient naturellement intérêt à suivre de près l'évolution de leur trésor, donc à obtenir l'établissement régulier d'états des comptes. Le document du Correr laisse entendre que ces extractus étaient délivrés au terme de chaque exercice annuel, mais on ne peut guère résoudre le problème de l'ancienneté et de la durée de cette pratique comptable. Nous ignorons beaucoup de l'organisation des finances royales aux XIII ${ }^{\mathrm{e}}-\mathrm{XIV}^{\mathrm{e}}$ s. et on peut avancer que la reddition de comptes sous forme d'extractus a été introduite avec les réformes fiscale et financière que Jacques $I^{\text {er }}$ engage avec le concours des officiers génois, après $1383^{19}$. Cette première hypothèse paraît logique, à défaut d'être tout à fait convaincante.

Comment expliquer, en effet, qu'au milieu du $\mathrm{XVI}^{\mathrm{e}}$ s., on reproduise uniquement l'extractus de 1412 ? Sachant la situation sereine de l'administration insulaire à partir de 1480, le fait que seul celui de 1412 ait été retrouvé induit que la disparition des archives des Lusignan avait commencé bien avant le sac de Nicosie par les Ottomans, en septembre 1570. Que l'extractus date du règne de Janus, et non de ceux de Jacques II (1461-1473) ou de Catherine Cornaro (14731489), laisse entrevoir la désorganisation des institutions centrales; une destruction partielle des archives intervient sans doute après le pillage du palais royal par les Mamelouks en juillet 1426, puis lors de la guerre civile entre Charlotte de Lusignan et Jacques le Bâtard (1458-1461), enfin pendant la révolte des nobles hostiles à Catherine Cornaro (été 1474). À chaque fois, le contrôle des registres fiscaux ou administratifs revêtait suffisamment d'importance pour qu'un parti ou l'autre tentât de les dissimuler ou de les détruire ${ }^{20}$.

Cependant, en 1524, quelque 80 volumes de Livres des Remembrances dorment encore dans les bureaux de l'administration vénitienne de Nicosie ${ }^{21}$; dès lors, comment admettre qu'aucun autre registre comptable n'ait été conservé avec ces précieux bilans? L'argument de la sauve-

$17 \mathrm{Cf}$., à titre de comparaison, les chiffres des comptes publiés par H. MORANVILLÉ, "Rapports à Philippe VI sur l'état de ses finances ", Bibliothèque de l'École des Chartes XLVIII (1887), p. 380-395; id., "Note sur des rapports financiers adressés à Philippe VI n, Bibliothèque de l'École des Chartes LIII (1892), p. 111-114.

$18 \mathrm{~J}$. VIARD, Journaux du Trésor de Philippe VI, suivis de l'Ordinarium Thesauri de 1338-1339 (1899), p. XXX-XXXII; R. FAWTIER, Comptes du Trésor 1296, 1316, 1384, 1477 (1930), p. VII-VIII; M. REY, Le domaine du roi et les finances extraordinaires sous Charles VI 1388-1413 (1965), p. 77-80.
19 MACHAIRAS, $\S 621$; F. BUSTRON, p. 352-353; AMADI, p. 495 ; STRAMBALDI, p. 258, 260.

20 En 1469, on ne pouvait retrouver le Livre des Remembrances de la Secrète de l'année 1460, ce que confirmait, un siècle plus tard, Zuan Zamberlan qui déplorait la disparition des livres des années $1458,1459,1460$ et 1461 : J. RICHARD, Le Livre des Remembrances, $n^{\circ} 220$; ASV, Capi del Consiglio dei Dieci, Dispacci di rettori e altre cariche, busta 290, c. $64-65$.

21 Correr, cod. Donà delle Rose 46, f. $10 \mathrm{v}$. 
garde aléatoire des archives du royaume franc ne peut tout expliquer, ce qui autorise une seconde hypothèse: l'extractus de 1412 serait une innovation dont l'origine pourrait être consécutive soit à l'éducation italienne de Janus, soit à l'installation de Charlotte de Bourbon; la filleule de Charles VI, qui - rappelons-le - était accompagnée d'une suite de soixante personnes, introduisit maints éléments de renouveau à la cour et renforça la culture franque dans une société gagnée par le métissage ${ }^{22}$. Lui attribuer une innovation d'ordre administratif paraît, a priori, surprenant, mais l'extractus de 1412 peut refléter, dans le domaine des techniques comptables, le goût marqué de Janus et Charlotte pour les modes françaises ${ }^{23}$.

Si l'on peut toujours espérer trouver dans les dépôts d'archives italiens d'autres documents financiers du royaume des Lusignan, le compte de 1412 reste un unicum, dont l'analyse permet de reconstituer l'agencement général des registres fiscaux de la Secrète. De toute évidence, les opérations du trésor étaient ventilées en deux états, celui des recettes, celui des dépenses, chacun étant divisé en rubriques définies selon l'origine ou la destination des fonds; en cela, le compte de 1412 reflète une organisation des registres commune à la plupart des trésoreries de l'Occident médiéval, les registres à partie double étant, alors, surtout répandus dans les comptabilités privées des marchands italiens ${ }^{24}$. Si d'autres registres ont existé, c'est-à-dire un journal du trésor, un livre de caisse, le document du Correr ne permet guère de l'assurer ${ }^{25}$.

L'extractus de 1412 se fonde certainement sur l'exploitation directe des livres de recettes et de dépenses de la Secrète, tenus durant une année fiscale qui commence au $1^{\text {er }}$ mars; les informations de l'extrait dévoilent donc un état des finances royales pour la période comprise entre le $1^{\text {er }}$ mars 1412 et le 28 février 1413. La méthode suivie pour consigner les articles n'offre aucune logique apparente; vraisemblablement, elle reproduit l'ordre adopté dans les livres. L'état des recettes dénombre 40 articles qui ne sont pas ventilés en grandes masses "budgétaires", à l'inverse de l'état des dépenses où les articles sont groupés en six rubriques approximatives. La comptabilité se montre donc plus détaillée pour les dépenses que pour les recettes. Rien ne permet d'affirmer que ce classement fut adopté par les Lusignan dès le XIII ${ }^{e}$ s., encore que le texte de l'allocution prononcée par les barons à Henri II, pour lui annoncer sa destitution en 1306, laisse supposer que les dépenses touchant l'hôtel du roi ont été organisées selon le modèle encore en vigueur en 1412, modèle presque universel chez les princes de l'Occident chrétien. Néanmoins, à la lecture de l'extractus, on comprend pourquoi ces comptes ont longtemps dérouté les officiers

22 Dans la liste des personnes composant la suite de Charlotte de Bourbon figure notamment le secrétaire Gillet cf. MACHAIRAS, $\S 642$.

23 On pense en effet davantage à une origine française qu'ita lienne, si l'on s'en tient à l'examen des comptabilités publiques; à Venise, le système fragmenté des administrations laisse une très grande indépendance financière à chaque office et entrave la compilation de bilans généraux; en revanche, à Gênes, les cartulaires des massari du milieu du $\mathrm{Xiv}^{\mathrm{e}} \mathrm{s}$. S'achèvent sur un bilan mais rien n'indique qu'un extractus ait été dressé à partir de ces bilans: R. CESSI, La regola zione delle entrate e delle spese (sec. XIII-XIV) (1925), p. v-X; T. ZERBI, Le origini della partita doppia (1952), p. 181-204. 24 F. MELIS, Aspetti della vita economica medievale (Studi nell'Archivio Datini di Prato) (1962), p. 391-403, 424-434.

25 À titre de comparaison, sept registres financiers du royaume de France sont connus à la même époque: le journal du Trésor, l'extractus Thesauri, le livre des recettes, le livre des dépenses, le livre du changeur, le compotus Thesauri et l'ordinarium Thesauri: J. VIARD, op. cit. (supra, n. 18), p. XXV-LIX; R. FAWTIER, op. cit. (supra, n. 18), p. VI-VIII; M. REY, op. cit. (supra, n. 18), p. 77-79. 
vénitiens; en 1480, Venise réformait ce système d'enregistrement en introduisant des livres ordinati alla Italiana (selon Florio Bustron), formule qui suggère l'introduction de registres à partie double ${ }^{26}$. Cette réforme transparaît à travers l'organisation du bilan de la camera daté de 1556 où les articles des recettes sont groupés en rubriques, caractère absent de l'extractus de 1412.

Comme dans tous les documents comptables du royaume, les sommes sont exprimées en besants (abrégé en b.), monnaie de compte depuis la fin du XIII ${ }^{e}$ s.; il est subdivisé en 24 caroubles ou carats. Dans les échanges quotidiens, le besant vaut 2 gros grands ou 4 gros petits ou 48 deniers $^{27}$. Quant à la valeur du besant par rapport au ducat vénitien, il convient d'apprécier sa parité en tenant compte du fait que sous le règne de Janus, le poids du gros diminue progressivement (passant de 4,90 à 4,40-4,55 gr.), sans qu'il soit possible de préciser la chronologie des altérations ${ }^{28}$. Les données qui peuvent être réunies montrent que le besant se déprécie sans arrêt depuis la fin du XIVe s., car sil faut fournir $41 / 4$ besants pour obtenir un ducat en 1395, il en faut 5 en 1404,4 ou 6 en $1410,53 / 4,6,6,6,6,8$ en $1423^{29}$.

\section{Les recettes}

L'état des recettes suit une organisation assez déroutante car la liste mélange des articles qui pouvaient aisément être rassemblés en rubriques; les revenus tirés des apauts de Cérines ou Pafos se trouvent divisés en deux postes sans raison apparente et d'autres exemples peuvent être produits. On ne saurait expliquer la logique interne du document, peut-être l'ordre chronologique de l'instauration des taxes ou des droits, voire les dates de leur encaissement mais ce ne sont que suppositions; privés des registres, nous sommes réduits aux conjectures. Les Vénitiens eux-mêmes avouaient leur désarroi face à ces livres d'une autre époque; on mesure le décalage entre les techniques comptables des secrétains du royaume franc et celles des camerlingues vénitiens en observant que les 40 articles de l'extractus de 1412 deviennent 14 rubriques dans le compte de 1556.

Ces 40 articles peuvent être regroupés en cinq grands chapitres: droits sur les fiefs, exploitation directe du domaine royal, fermes (apauts) et locations, imposition extraordinaire, redevances diverses. Leur montant s'établit de la manière suivante:

26 G. GRIVAud, "Ordine della Secreta di Cipro", p. 566.58; cette réforme intervient en 1480 , selon le syndic Jacopo Semitecolo (1530): Marciana, cod. ital. cl. VII, $n^{\circ} 1663$ (=9612), f. $21 \mathrm{v}-22$ r. Notons cependant que le registre à partie double est utilisé dans les comptabilités de particuliers, ainsi en 1423 : J. RICHARD, Documents chypriotes, p. 22-30. 27 J. RICHARD, Documents chypriotes, p. 17-18; D. M. METCALF, Coinage of the Crusades and the Latin East in the Ashmo- lean Museum Oxford (1995), p. 199-200.

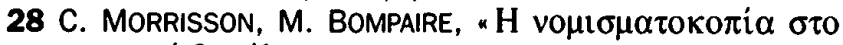
$\mu \varepsilon \sigma \alpha \iota \omega \mathrm{Vl \kappa ó} \beta \alpha \sigma i ́ \lambda \varepsilon l o ~(1411-1489)$ ", IK V, p. 1477.

29 L. DE MAS-LATRIE, Histoire II, p. 424-425; J.-C. HOCQUET, op. cit. (supra, n. 1), I, p. 199; J. RICHARD, Documents chypriotes, p. 24 ; P. SPUFFORD, Handbook of medieval exchange (1986), p. 298-299; C. MORRISSON, M. BOMPAIRE, art. cit., p. 1477. 
TABLEAU 1

Les recettes du royaume de Chypre en 1412 (année fiscale)

\begin{tabular}{llll} 
Domaine royal & b. & $361519 / 19$ & $32,37 \%$ \\
\hline Droits sur les fiefs & b. & $261759 / 12$ & 23,44 \\
\hline Extraordinaire & b. & 246241 & 22,05 \\
\hline Apauts et locations & b. & $195262 / 18$ & 17,48 \\
\hline Divers & b. & $51743 / 9$ & 4,63 \\
\hline Total & b. $1116526 / 10$ & \\
\hline
\end{tabular}

Plusieurs remarques sur cet état des recettes. En premier lieu, on constate la solidité des revenus du domaine royal qui représentent près du tiers de l'encaisse annuelle. L'exploitation en faire-valoir direct des villages fournit à elle seule près de 250000 besants, soit presque le quart des recettes globales, bien plus que les fermes et locations, qui laissent à peine 200000 besants $(17,48 \%)$. La juxtaposition de ces chiffres illustre combien Janus et, sans doute, Jacques I $^{\text {er }}$ avant lui, ont appliqué une politique de gestion directe de la regale. En 1412, on peut estimer que les trois quarts des villages du domaine sont gérés par les officiers du roi dans le cadre des bailliages, contre un quart, environ, cédé à bail. À l'évidence, les derniers Lusignan ont renforcé leur contrôle sur l'exploitation des ressources agricoles, considérant l'économie rurale comme la source essentielle de leurs recettes.

Le produit des fermes et loyers de la regale sont aux deux tiers composés d'impôts indirects frappant la circulation des denrées, leur négoce et diverses transactions; seul le comerc, taxe de faible valeur perçue sur le pesage de certaines marchandises, est levé directement par les officiers du roi. Le compte de 1412 montre la part réduite des revenus tirés du commerce intérieur, sans doute trop irréguliers du fait des crises causées par les épidémies et les nuées de sauterelles. Les choix de Jacques $\mathrm{I}^{\text {er }}$ et de Janus illustrent une économie de repli, qui tourne le dos à ces vastes échanges commerciaux à l'échelle méditerranéenne, ceux dont les bénéfices avaient provoqué la richesse légendaire de Famagouste. Mais pouvait-il en être autrement, eu égard à la conjoncture déprimée du dernier quart du XIV $s$. et de la première décennie $d u \mathrm{XV}^{\mathrm{e}}$, alors que le monopole du commerce de l'île était passé aux mains des Génois? ${ }^{30}$

Une autre évolution importante dévoilée par l'extrait de 1412 concerne la fiscalisation des obligations féodales. Peter W. Edbury avait observé que jusqu’à la fin du XIV ${ }^{e}$ s. les obligations liées au servise de cors étaient respectées, c'est-à-dire assurées en personne ${ }^{31}$. L'évolution à l'époque de Janus s'avère très nette, puisque le rachat des défauts de service fournit plus de $12 \%$ des

30 R. S. LOPEZ, "The Trade of Medieval Europe: the South ", in M. POSTAN, E. E. RICH (éds), The Cambridge Economic History of Europe, II. Trade and Industry in the Middle Ages (1952), p. 338-353; J. HEERS, "Il commercio nel Mediterraneo alla fine del sec. XIV e nei primi anni del XV", Archivio storico italiano 113 (1955), p. 202-209; J. DAY, Les douanes de Gênes 1376-1377 (1963), I, p. XXXV;
M. BALARD, La Romanie génoise ( $x \|^{*}$-début du $x v^{e}$ siècle) (1978), II, p. 683; R. FosSIER, Le Moyen Age, III. Le temps des crises $1250-1520$ (1983), p. 56-58; F. C. LANE, Venise, une république maritime (1985), p. 46-50; J. DAY, The Medieval Market Economy (1987), p. 203-211.

31 P. W. EDBURY, "Feudal obligations in Latin East ", Byzantion XLVII (1977), p. 335-337. 
recettes du trésor (134 104 besants) ; cette mesure, engagée beaucoup plus tôt en Occident, fournissait les liquidités indispensables à l'entretien de soldats soudoyés. Les mobiles financiers n'expliquent pas tout cependant car, après 1347, les lignages francs sont frappés par les épidémies; nombre de fiefs sont tenus par des mineurs qui, jusquà leur majorité, ont la possibilité légale de racheter leur service; d'autres fiefs deviennent vacants ou tombent en déshérence, ce qui provoque leur retour à la regale ${ }^{32}$. Si nous ignorons la liste exacte des services auxquels correspondent les rachats, la somme encaissée en 1412 équivaut à quelque 167 services de chevaliers ${ }^{33}$.

Enfin, il faut observer le poids de l'imposition extraordinaire dans le compte de 1412, puisque la dîme royale fournit plus d'un cinquième des recettes globales; ces chiffres démontrent l'efficacité de l'aide établie par Jacques $\mathrm{I}^{\text {er }}$, à partir de $1385^{34}$. Cette taxe, qui pèse sur tous les revenus - y compris la dîme ecclésiastique -, suivait les fluctuations des rentes; comme l'année fiscale 1412 paraît échapper aux catastrophes naturelles, on peut accepter la somme de 134000 besants comme le produit moyen de l'impôt. Remarquons enfin que, si Jacques Ier avait justifié le recours à l'extraordinaire par la dette aux Génois, entre janvier 1413 et août 1414, les massarii de la vieille mahone de Chypre encaissent 171712 besants à partir de versements établis sur d'autres recettes que la dîme royale (grande gabelle de la porte de Nicosie, gabelle sur l'estampillage des camelots $)^{35}$. Force est de constater que la dîme royale avait rapidement été détournée de sa destination première; lourde et imposée à la demande des Génois, la dîme royale ne pouvait, en conséquence, que rencontrer une impopularité croissante ${ }^{36}$. Néanmoins, le produit de la dîme royale assure de solides revenus au trésor; associé aux sommes obtenues par les rachats de service, il apparaît que plus du tiers des recettes de Janus est formé des revenus de la nouvelle fiscalité, entendons celle instituée après 1385 .

\section{Les dépenses}

Plus clair que le chapitre des recettes, celui des dépenses se révèle mieux structuré, même si le plan des rubriques énoncé en début de chapitre n'est pas respecté. Là encore, de singulières incohérences peuvent être relevées, puisque les frais occasionnés par l'entretien des bains royaux sont divisés en trois postes, sans raison apparente, si bien que la rubrique de l'hôtel du roi comprend finalement 24 articles. On réorganisera les rubriques de la manière suivante:

32 C'est ainsi que semblent pouvoir être interprétés les deux articles: Per feudi de cavallari, et altri che sono in la real, Per feudi pervenuti in la real per 5 anni. Plus généralement, sur le processus de fiscalisation du fief, on consultera J. RICHARD, "Le régime des fiefs à Chypre entre service armé

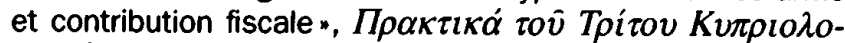

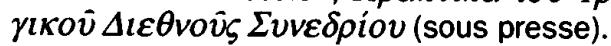

33 Puisque le rachat d'un fief de chevalier se monte à 800 besants (cf. les références citées plus bas, n. 73 de l'ap- pendice). Sur la base des documents statistiques vénitiens de c. 1521, publiés par L. DE MAS-LATRIE, Histoire III, p. 504513 , on peut avancer que sur les 900 villages de l'île, 260 appartiennent au domaine royal; les autres casaux étant distribués sous forme de fiefs, 167 services de chevaliers représentent environ un quart des dotations.

34 MACHAIRAS, § 618, 621; F. BUSTRON, p. 352-353, 463.

35 C. OTTEN-FrOUX, art. cit. (supra, n. 4), p. 63, 67.

36 MACHAIRAS, § 623. 
TABLEAU 2

Les dépenses du royaume de Chypre en 1412 (année fiscale)

\begin{tabular}{lll} 
Assignations & b. $240481 / 21$ & $71,34 \%$ \\
\hline Hôtel du roi & b. $52461 / 2$ & 15,56 \\
\hline Dépenses privées $*$ & b. $23588 / 201 / 2$ & 7,00 \\
\hline Pensions à la famille du roi & b. $20536 / 22$ & 6,09 \\
\hline Total & b. $337069 / 15$ & \\
\hline
\end{tabular}

* Du roi, de la reine et des enfants naturels de Janus

L'essentiel des dépenses $(71,34 \%)$ se trouve consacré aux assignations versées aux fieffés et aux soldes; on mesure ainsi le coût de la fidélité des nobles et la charge que représente l'entretien des troupes au lendemain des guerres génoises. À cette masse financière importante, il convient d'ajouter d'autres postes insérés dans les articles de l'hôtel royal pour apprécier le total des charges fixes affectées au personnel qui dépend du roi ; 9600 besants sont versés en soldes et 32007 consacrés aux rémunérations du personnel de cour, composé de domestiques, de secrétains et d'officiers mineurs. Au total, plus de $83 \%$ des dépenses de l'exercice 1412 sont affectés aux rentes, assignations et salaires.

Les dépenses privées de la famille royale viennent, pour leur part, informer sur le train de vie des Lusignan. Comme attendu, la hiérarchie des sommes allouées consacre la fortune du roi (14754 b.) sur celles de son épouse (7068 b.), de la reine mère $(5471 \mathrm{~b}$.), des six frères et sœurs de Janus (entre 2572 et 2489 b.); 1762 besants sont enfin attribués aux trois enfants naturels du roi, âgés de 2 à 6 ans, somme qui correspond sans doute à l'entretien des nourrices. La famille royale grève le budget de 44125 besants et $181 / 2$ caroubles, soit plus de $13 \%$. Notons que les pensions sont versées exclusivement en numéraire pour les parents de Janus alors que le roi, surtout, et sa femme, dans une moindre mesure, reçoivent des fournitures en nature pour une valeur d'environ 11500 besants. La consommation de $900 \mathrm{~kg}$ de cire pour l'éclairage personnel du roi donne une image assez étonnante de la dimension des appartements du palais, ou, plus vraisemblablement, des salles des diverses résidences royales.

La principale surprise tirée de l'examen des dépenses affectées à l'hôtel du roi tient en la faible part des frais d'administration. Si l'on excepte du total les rémunérations du personnel, 10854 besants seulement leur sont consacrés; sur cette somme, les charges attribuées au fonctionnement de la maison s'avèrent d'une incroyable modestie : 220 besants pour les frais de gestion administrative et de chancellerie (papier, encres), 250 pour la paneterie, 300 pour l'achat de bois. Des sommes dix fois inférieures à celles destinées au train de vie de Janus: 3131 besants pour la fauconnerie, 1200 pour l'écurie, 842 pour les bains, 282 pour la chapelle, 111 pour le chenil, dépenses auxquelles il convient d'ajouter les largesses aux chanteurs et médecins ( 2000 besants). À travers cette répartition, se dessine le goût de Janus pour une vie de cour teintée de faste. 


\section{Les silences du compte}

Aussi dense soit-il, le document du Correr ne présente pas un état complet des comptes du royaume de Chypre pour l'année fiscale 1412. Ainsi, le copiste vénitien précise, à juste titre, l'absence des revenus tirés des salines et de Famagouste. Bien d'autres silences méritent encore d'être signalés.

Depuis longtemps, la production de sel des lacs de San Lazzaro, à Larnaca, était passée sous le contrôle effectif des Vénitiens; dès le règne de Pierre II, les emprunts à Federico Corner étaient assis sur des lettres de paiement en sel, mesure qui équivalait à abandonner aux Vénitiens le monopole du commerce de la production chypriote. En 1399, alors que les dettes étaient presque éteintes, Giovanni Corner exerce une pression suffisante pour obtenir le renouvellement de ses privilèges pendant une durée de dix ans; cette situation se prolonge dans les années qui suivent, même si Janus tente de spéculer sur les prix et mesures pour augmenter ses profits ${ }^{37}$. Après le traité de paix extorqué par Gênes en 1403, le roi est contraint d'emprunter de nouvelles sommes d'argent aux Vénitiens, remboursées partie en comptant, partie en sel; de fait, le roi leur abandonne l'exploitation de la production saline. En 1412, la récolte de sel se trouve donc sous contrôle vénitien et - fort logiquement — l'extractus n'enregistre aucune somme versée au trésor par les Corner. Si le volume exact de la récolte de sel de 1412 demeure inconnu, en 1393, la production de sel atteignait 260000 mozetti chypriotes, écoulés pour une somme d'environ 234000 besants (au prix fixé, en 1399 , de 90 besants le cent de mozetti) ${ }^{38}$. Un revenu comparable à celui tiré de la dîme royale échappait donc au trésor de Janus pour gagner directement les caisses des patriciens vénitiens.

Que les Corner aient tiré profit des prêts accordés à Janus résultait en grande partie des charges que les Lusignan devaient honorer vis-à-vis de Gênes, l'autre puissance se nourrissant de la faiblesse maritime et militaire du dernier royaume franc d'Orient. Les recettes de Famagouste ne figurent pas dans le compte de 1412 puisque, par le traité du 19 février 1383, la ville et le territoire compris dans un périmètre de deux lieues à la ronde sont aux mains des Génois. Les clauses du traité prévoyaient encore que tout le commerce d'exportation de l'île devait transiter par le port de Famagouste et que les taxes levées à cette occasion restaient acquises à Gênes. Composé principalement des revenus du comerchium et de la gabelle (octroi), le montant exact des recettes de l'année 1412 n'a pas été conservé dans les archives génoises; on peut l'évaluer à plus de 100000 besants à partir des données rassemblées par Michel Balard (112998 besants en $1408,127406$ besants en 1443$)^{39}$.

37 G. LUZzATTO, "Sindacati e carteli nel commercio veneziano nei secoli XIII e XIV", Rivista di storia economica I (1936), p. 62-66, repris dans id., Studi di storia economica veneziana (1954), p. 197-199; J. RICHARD, "Chypre du protectorat à la domination vénitienne ", in A. PERTUSI (éd.), Venezia e il Levante fino al secolo xV (1973), I/2, p. 660-
663, repris dans id., Croisés, missionnaires et voyageurs (1983), étude $n^{\circ}$ XII; J.-C. HOCQUET, op. cit. (supra, n. 1), I, p. $198-202$.

38 Ibid., I, p. 199-200.

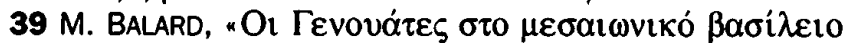

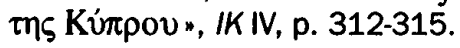


D'autres silences traversent le compte de 1412, tant au titre des entrées qu'à celui des sorties. Ainsi, deux impôts ne figurent pas dans les articles des recettes: la maréchaussée, redevance d'origine byzantine qui frappe le bétail des paysans libres, et la mète du sel, impôt extraordinaire établi par Jacques I ${ }^{\text {er }}$, vers 1395, qui contraint tous les paysans à verser un besant pour acquérir un muid de sel. Ces deux redevances ne paraissent pas levées et encaissées par la Secrète mais par des officiers indépendants; en remettaient-ils le produit au trésor ou bien constituaient-ils des caisses autonomes que le roi gérait à sa convenance? Les relations entre les différents services de l'administration fiscale nous demeurant inconnues, il paraît bien imprudent de répondre; néanmoins, à la lecture des actes du Livre des Remembrances de l'année 1468, la seconde hypothèse semble plus proche de la réalité ${ }^{40}$. Une confirmation tardive peut être décelée à l'époque vénitienne, puisque la balance des comptes de l'année 1559 enregistre à part le poste "da marzasone et metta del sale" "41.

Une autre source de revenus n'apparaît pas dans l'extractus, à savoir le produit du rachat des gardes des côtes versé par les francomates du domaine royal. S'il reste malaisé d'évaluer la fréquence de ce rachat au début du XVe s., on sait que l'habitude de la commutation du service du guet s'est généralisée sous Jacques II ; alors, les sommes perçues alimentent des caisses régionales (une par contrée) sur lesquelles le roi rembourse diverses créances; là encore, on constate l'existence de caisse autonome non intégrée au trésor et dont les comptes ne sont pas inclus à l'extractus de $1412^{42}$.

On observe, enfin, que le compte de 1412 ne mentionne aucune recette au titre des butins des expéditions maritimes ${ }^{43}$ et, surtout, à celui du seigneuriage, alors que la frappe de la monnaie constitue un monopole royal depuis Guy de Lusignan ${ }^{44}$. Grâce à Francesco Balducci Pegolotti, facteur des Bardi à Famagouste dans les années 1330, on connaît assez précisément les opérations de transformation des monnaies d'argent pour que les marchands étrangers obtiennent des gros, les seules pièces ayant cours sur les marchés de l'île. Pegolotti détaille le montant des taxes levées par l'hôtel des monnaies de Famagouste $(2,76 \%)$ et assure que la frappe des gros est affermée ${ }^{45}$. Par ailleurs, les études de numismatique de D. M. Metcalf mettent en évidence l'existence d'un second atelier de frappe, fort probablement situé à Nicosie, doté sans doute d'une administration

40 Sur la maréchaussée: J. RICHARD, "Le casal de Psimolofo et la vie rurale en Chypre au XIVe s. ", MEFRM LIX (1947), p. 145, repris dans id., Les relations entre l'Orient et l'Occident au Moyen Âge (1977), étude $n^{\circ} \mathrm{V}$; id., "Agricultural conditions in the Crusader States * in K. SETTON (éd.), A History of the Crusades (1985), V, p. 272; J. RICHARD, Le Livre des Remembrances, $n^{\text {os }} 21,33,78,97,143,145$, 168; G. GRIVAUD, "Ordine della Secreta di Cipro ", p. 581; il reste, cependant, à prouver que cette maréchaussée, díme sur le bétail, relève de l'office de la maréchaussée, bureau chargé de l'enregistrement des hommages: G. Grivaud, "Ordine della Secreta di Cipron, p. 563.49-51 et n. 193. Sur la mète du sel: MACHAIRAS, § 625 ; J. RICHARD, Le Livre des
Remembrances, p. XVI, nos 30, 78, 101, 122.

41 Marciana, cod. ital. cl. VI 80 (=5767), f. 180r.

$42 \mathrm{~J}$. RICHARD, Le Livre des Remembrances, $n^{\circ 5} 8,48,122$.

43 Le produit des butins, qui ne saurait former un revenu régulier, était peutêtre administré indépendamment par l'office de l'amiral, comme le suggère Jean Richard (communication personnelle).

44 C. MORRISSON, M. BOMPaIRE, art. cit. (supra, n. 28), p. 1469.

45 F. BALDUCCI PEgolotTI, La pratica della mercatura (éd. A. EVANS) (1936), p. 82-83 et les commentaires de C. MORRISSON, M. BOMPAIRE, art. cit., p. 1470-1472. 
spécifique ${ }^{46}$. Ces informations concordent pour assurer que la gestion des stocks d'argent n'appartient ni à la Secrète, ni à son trésor, mais à deux bureaux autonomes, baillés à ferme. Après 1374, la situation se simplifie puisque Famagouste passe sous contrôle génois et que le port doit, d'après les traités, absorber tout le commerce de l'île. Ne pouvant utiliser la zecca de Famagouste, Jacques $\mathrm{I}^{\text {er }}$ et Janus frappent à Nicosie et, peut-être, à Limassol. Or les découvertes monétaires assurent que Janus émet des gros grands, des gros petits, des deniers, sans qu'il soit possible de comprendre d'où proviennent les métaux (les fameux butins?) ${ }^{47}$; Machairas souligne encore que Janus frappe un sizain de billon ( 6 deniers) pour financer sa guerre contre Famagouste, entre 1403 et $1406^{48}$. Janus spécule sur le poids des monnaies pour tenter de régler ses dettes, notamment en réduisant le poids du gros de 4,90 à 4,40-4,55 gr. mais cette altération n'apparaît pas dans le compte de 1412; de la même manière, les activités monétaires de Jacques II ne figurent pas dans le Livre des apauts inclus aux Livres des Remembrances de l'année fiscale 1468. Assurément, la monnaie relevait d'un autre office: jamais les Livres de la Secrète ne dévoilent la politique monétaire des Lusignan. On ne peut donc estimer les revenus que la couronne tirait de son monopole sur la monnaie au début du XVes.

Au chapitre des dépenses, les soldes versées aux cavallari et stipendiati couvrent-elles la totalité des gages versés aux soldats? Nous sommes bien en peine de l'affirmer, étant donné notre méconnaissance des contingents de l'armée chypriote en période de paix; en 1403, Janus sollicitait un emprunt auprès de Venise pour payer les gens d'armes qu'il escomptait enrôler à Venise afin de reprendre Famagouste ${ }^{49}$; rien n'indique qu'en 1412 , ses besoins militaires l'aient contraint à renouveler de tels emprunts. En revanche, on s'étonne de relever l'absence de dépenses d'ordre stratégique: entretien des enceintes des villes, des châteaux et, surtout, de la marine, si active selon Léontios Machairas; là encore, le fruit des pillages pouvait être directement versé à l'entretien de la galée et de la galiote que Janus armait. Par ailleurs, sachant l'éclat de la cour de Janus et Charlotte, comment accepter le silence sur les frais d'entretien des manoirs de plaisance?

Enfin, au-delà de toutes ces incertitudes, nulle part n'apparaissent les dépenses diplomatiques, notamment les sommes remboursées à Gênes. Une partie des 108899 besants et 21 caroubles versés aux massari de Famagouste entre juillet 1411 et janvier 1413 aurait dû figurer dans le compte de $1412^{50}$. D'autres dettes, aux Vénitiens cette fois, n'étaient pas éteintes, provoquant les plaintes réitérées du Sénat en juillet 1411, août 1412, août 1414; la plupart d'entre elles devant être satisfaites au moyen d'assignations sur les récoltes de sucre, il eût été logique d'en trouver trace dans le compte de $1412^{51}$.

Ce qui est à retenir des omissions de l'extractus est que le budget préparé par la Secrète ne prend pas en compte toutes les sources de revenus et toutes les charges qui incombent à la cou-

46 D. M. METCALF, op. cit. (supra, n. 27), p. 203-206.

47 Id., op. cit., p. 213-215.

48 MACHAIRAS, § 634; ASV, Senato Misti, reg. 46, f. 101, reg. 50, f. 146v; D. M. METCALF, op. cit., p. 215.
49 L. DE MAS-LATRIE, Histoire II, p. 456.

50 C. OTTEN-FrouX, art. cit. (supra, n. 4), p. 72.

51 ASV, Senato Misti, reg. 50, f. 145r-147r; L. DE MASLATRIE, Histoire II, p. 457-458. 
ronne. Ceci mène à interroger les offices parallèles qui pouvaient exister au sein des institutions de la royauté chypriote. À l'époque de Jacques II, le roi n'hésite pas à asseoir rentes ou assignations sur le produit de la maréchaussée ou de la mète du sel sans attendre leur passage par le trésor central. Janus et ses prédécesseurs agissaient-ils déjà de la sorte? En quel cas, par-delà l'état des comptes établi par la Secrète devait exister un "budget " plus général englobant toutes les recettes et dépenses de la couronne. L'extractus de 1412 ne reflète que la partie centrale du budget du royaume.

En conséquence, l'excédent d'environ 780000 besants ne représente qu'une évaluation globale de l'actif du trésor. Les recettes de la couronne se situent au-delà des chiffres rapportés par le document; on peut ajouter aux 1116526 b.: les ventes de sel (200000 b.), la recette de la mète du sel (au moins 70000 b.52), celle de la maréchaussée (environ 70000 b.53). Sans compter le butin acquis aux dépens des Mamelouks, les recettes du seigneuriage, le rachat des gardes des francomates et les revenus de Famagouste, le revenu global de la couronne dépasse le million et demi de besants, revenu qui paraît correspondre à celui d'une année normale, dégagée des effets d'épidémies ou de récoltes calamiteuses.

Il reste, par contre, bien impossible de déterminer le volume des dépenses, donc d'apprécier la balance entre les entrées et les sorties. Dans tous les cas, Janus vit aisément du sien et possède les ressources nécessaires pour éteindre la dette génoise, sachant, au moment opportun, puiser dans le trésor lorsqu'il nourrit des ambitions diplomatiques; ainsi, en 1401, il promet une dot de 130000 ducats à sa sœur Marie qui épouse Ladislas, roi de $\mathrm{Naples}^{54}$; en 1403, il offre 25000 ducats au maréchal Boucicault ${ }^{55}$; en 1410-1412, Janus obtient pour son fils naturel Aloysius la commende de la Grande Commanderie de Chypre, au terme d'un marchandage avec Jean XXIII qui lui coûte 6000 ducats $^{56}$. La lenteur des Lusignan à satisfaire les exigences génoises relevait d'une mauvaise volonté politique évidente; pendant ses quinze années de règne, Jacques $I^{\text {er }}$ construisit ou reconstruisit le château de Sigouris, le palais de La Cava, les manoirs d'Aradippou et de Potamia, résidences que Janus lui-même embellit ${ }^{57}$; avec Charlotte de Bourbon, Janus fit élever la chapelle de Pyrga et entretint à sa cour un groupe de musiciens venus d'Italie et de France ${ }^{58}$.

52 À raison d'une population paysanne estimée à 70000 âmes (hypothèse basse); Jacques I $^{\text {er }}$ en tira 80000 b. en 1395 , selon MACHAIRAS, $\S 625$.

53 Estimation contestable, on en convient, induite par le fait que Jacques II rembourse un prêt de 100 ducats, moitié sur la mète du sel et moitié sur la maréchaussée de la contrée de Pentageia en 1468; en 1559, les officiers vénitiens comptabilisent ensemble ces deux recettes, qui, sur l'ensemble du regno, rapportent 12324 ducats (= 123240 b.): J. RICHARD, Le Livre des Remembrances, no 78; Marciana, cod. ital. cl. VI 80 (= 5767), f. 180r.

54 L. DE MAS-LATRIE, Histoire II, p. 465-466, 477-480.

55 Le livre des fais du bon messire Jehan le Maingre, dit Bouciquaut (éd. D. LALANDE) (1985), p. 231.

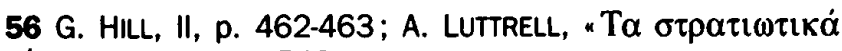
$\tau \dot{\alpha} \gamma \mu \alpha \tau \alpha$ ", IK IV, p. 749.

57 C. ENLART, L'art gothique et la Renaissance en Chypre (1899), II, p. 556-557, 658-661; G. JEFFERY, A Description of the Historic Monuments of Cyprus (1918), p. 181, 203 204 ; N. CHRISTOFIDOU, art. cit. (supra, n. 3), p. 165-171.

58 C. ENLART, op. cit., I, p. 428-439; G. JEFFERY, op. cit., p. $340-343$; A. W. CARR, "Byzantines and Italians on Cyprus: Images of Art ", Dumbarton Oaks Papers 49 (1995), p. 347 348; U. GÜNTHER, L. FINSCHER (êds), The Cypriot-French Repertory of the Manuscript Torino J.Il.9 (1995); Music from the Court of King Janus at Nicosia, P. VAN NEVEL, Huelgas Ensemble, 1993 (Sony SK 53976). 


\section{Les finances du royaume de Chypre en 1412}

L'extractus de 1412 procure une image assez précise des sommes dont dispose la couronne à une époque charnière de son histoire; Janus commence à se dégager de la dette génoise et n’a pas subi l'humiliation de Choirokitia. De prime abord, on accepte 1412 comme une année représentative de la phase de répit, voire de redressement, que connaissent les affaires du royaume entre 1411 et 1424.

Cette proposition ne peut être appréciée à partir de l'examen des revenus des Lusignan aux époques précédentes, car les renseignements, lacunaires, fragmentaires, restent sujets à caution. Ainsi, une Continuation de Guillaume de Tyr assure qu'à la mort d'Aimery (1205), les revenus de la couronne atteignent 200000 besants, mais une autre version du texte porte le chiffre à 300000 besants $^{59}$ ! Léontios Machairas fournit lui aussi des données chiffrées, pour l'année 1306, lorsque Henri II doit accepter sa déposition; Henri obtient une rente annuelle de 100000 besants pour ses dépenses personnelles et celles de son hôtel; son neveu Hugues, futur Hugues IV, reçoit pour sa part une rente de 10000 besants, la reine mère 20000 , les sœurs du roi 10000 , leur tante 20000 et ses filles $4000^{60}$. Ces données montrent que les Lusignan disposent au début du $\mathrm{XIV}^{\mathrm{e}} \mathrm{s}$. de revenus bien supérieurs à ceux de 1412, mais les dépenses de Janus et de son hôtel représenteraient environ les deux tiers de celles d'Henri, étant entendu que le roi destitué disposait d'une partie seulement des ressources de la couronne et que l'essentiel des revenus entrait dans les caisses d'Amaury. Le conditionnel reste, cependant, de rigueur tant les comparaisons ne valent que lorsqu'elles concernent des postes rigoureusement identiques. Pour l'instant, il n'est guère possible d'apprécier, de manière plus correcte, la place du budget de 1412 par rapport aux finances du royaume franc des XIII et $\mathrm{XIV}^{\mathrm{e}} \mathrm{s}$.

En revanche, les données se révèlent plus précises à la fin $\mathrm{du} \mathrm{XV}^{\mathrm{e}}$ s., quand les officiers vénitiens établissent des estimations des revenus du domaine royal qu'ils gèrent à partir de 1489 . À l'époque de Jacques II (1461-1473), les revenus de la couronne sont compris entre 80000 et 90000 ducats (entre 560000 et 630000 besants) ${ }^{61}$. Ces évaluations trouvent confirmation avec un des premiers bilans de la colonie vénitienne; en 1491, les camerlingues encaissent 75228 ducats ( 526596 b.), en déboursent 69494 (486458 b.), pour obtenir un solde positif de 5734 ducats $(40138 \text { b. })^{62}$. En s'en tenant à la simple observation des recettes, celles de Janus se révèlent presque trois fois supérieures à celles de 1491 ; il faut attendre 1558, lorsque Chypre connaît une période d'expansion démographique et économique, pour que les recettes retrouvent

59 Recueil des Historiens des Croisades, Historiens occidentaux (1859), II, p. 190-191; La Continuation de Guillaume de Tyr (1184-1197) (éd. M. R. MORGAN) (1982), p. 136-138.

60 MACHAIRAS, § 55, confirmé par le texte officiel de l'accord passé entre Henri II et son frère Amaury (hormis la mention des 20000 b. de la reine mère qui n'apparaissent pas): L. DE MAS-LATRIE, "Texte officiel de l'allocution adres- sée par les barons de Chypre au roi Henri II de Lusignan pour lui notifier sa déchéance", Revue des questions historiques XLIII (1888), p. 538-539.

61 ASV, Senato Mar, reg. 12, f. 70r; le texte précise la parité entre les monnaies au taux de 1 ducat $=7$ besants. 62 F. THIRIET, La Romanie vénitienne au Moyen Âge. Le développement et l'exploitation du domaine colonial vénitien (XIF. $x v^{e}$ siècles) (1959), p. 436. 
le niveau atteint sous Janus ${ }^{63}$. Ces chiffres, plus cohérents que ceux disponibles pour les XIII $\mathrm{XIV}^{\mathrm{e}}$ s., assurent que le règne de Janus, au moins jusqu'en 1424, ne s'identifie pas à la période de déclin trop souvent décrite.

Une appréciation similaire peut être tirée de l'examen du poids financier du royaume de Chypre à l'échelle de l'Occident; les estimations des revenus (intrade) des princes chrétiens qui circulent à Venise en 1423, quelles que puissent être les réserves émises à leur sujet ${ }^{64}$, fournissent d'utiles éléments de réflexion, d'autant plus que Chypre ne figure pas dans ces listes ${ }^{65}$. Si l'on accepte le ducat équivalant à 6 besants, le revenu de la couronne chypriote s'établit à quelque 250000 ducats, en 1412; à l'aune des princes d'Occident, le roi de Chypre possède des revenus comparables à ceux du roi du Portugal (1410: 200000 d.) ou du comte de Bretagne (1414: $200000 \mathrm{~d}$.), mais bien inférieurs à ceux des grandes monarchies: Espagne (1410:3 millions de ducats), Bourgogne (1400: 3 millions de ducats), France ou Angleterre (1410:2 millions de ducats).

La liste vénitienne devient beaucoup prolixe pour l'année 1423. Nous ne possédons pas le bilan du royaume de Chypre de cette année-là, mais les annales et les chroniques ne relevant aucune catastrophe aux conséquences dramatiques durant la période $1413-1423^{66}$, on peut avancer que Janus maintient ses ressources au niveau de 1412, s'il ne les consolide, les destructions des Mamelouks n'intervenant qu'à partir de l'été 1424. Cette stabilité supposée des revenus de Janus contraste avec l'effondrement des trésors des princes chrétiens, souvent engagés en de longues guerres; en 1423, la couronne de Chypre possède des revenus d'un niveau comparable à ceux de la Toscane ou de Bologne, supérieurs (de 50000 à 100000 ducats) à ceux de Bretagne, de Savoie, de Ferrare et de... Gênes ${ }^{67}$; Venise et son Dogado, en temps de paix, déclaraient 1,1 million de ducats, auxquels s'ajoutaient 464000 ducats de revenus en Terre Ferme et 376000 ducats dans le Dominio da mar.

Sans attacher une valeur absolue à ces chiffres, ils confirment les raisons qui poussent les Italiens à suivre de près les affaires chypriotes, les perspectives économiques recoupant les mobiles d'expansion politique; Gênes ne cesse d'accentuer son exploitation financière sur le royaume; des 8 millions de besants réclamés en 1374, réduits à moins de 4 millions en 1387, Gênes en obtient presque 3,5 millions ${ }^{68}$. Au XV $\mathrm{XV}^{\mathrm{e}}$ s., la maison de Savoie réalise deux unions avec les Lusignan; la première, en 1432, conduit Anne, fille de Janus, à Chambéry, avec une dot de 100000 ducats $^{69}$; la seconde, en 1458, permet aux princes de Savoie d'acquérir les couronnes de

63 B. ARBEL, art. cit. (supra, n. 10), p. 484.

$64 \mathrm{~F}$. BRAUDEL, La Méditerranée et le monde méditerranéen à l'époque de Philippe II (1979) ${ }^{4}$, II, p. 28; id., Civilisation matérielle, économie et capitalisme $x v^{e}-x V I I F$ siècle (1979), III, p. 97-99.

65 F. BESTA (éd.), Bilanci generali della Repubblica di Venezia (1912), I/I, p. 98-99.

66 Signalons cependant un retour de la peste en 1419 et/ou 1421: AMADI, p. 499; STRAMBALDI, p. 267.
67 Pour Gênes, les chiffres du budget ne signifient pas grand-chose dans la mesure où les intérêts privés avaient confisqué à leur profit une part importante des revenus publics : F. BRAUDEL, op. cit., III, p. 98.

68 C. OTTEN-FrouX, art. cit. (supra, n. 4), p. 66, 69.

69 F. DE CARIA, D. TAVERNA, "Les Lusignan et la maison de Savoie: le mariage entre Louis II et Anne de Lusignan de Chypre (1432-1462)" in C. MUTAFIAN (éd.), op. cit. (supra, n. 1), p. 118. 
Chypre et de Jérusalem. Quant à Venise, elle augmente sa puissance financière en annexant à son domaine, en 1489, une île dont les revenus demeurent trois fois supérieurs à ceux de la Crète en $1509^{70}$.

En d'autres termes, le concept de décadence économique du royaume de Chypre au XVe $s$. mérite d'être nuancé. Les règnes d'Henri II et d'Hugues IV, avec leur incontestable opulence, ont été décrits comme un âge d'or par les chroniqueurs du $\mathrm{XV}^{\mathbb{e}} \mathrm{s}$., des chroniqueurs spectateurs de désastres militaires, de villages décimés par les épidémies, de campagnes ruinées par les mauvaises récoltes, de ports délaissés par les marchands. Chypre subit les catastrophes que le Proche-Orient et l'Occident connaissent à la même époque; à ce titre, on ne saurait nier la phase de repli démographique et économique qui frappe l'île mais, replacée dans son contexte méditerranéen, cette crise oblige le royaume de Chypre à privilégier des sources de revenus qui dépendent de ses capacités productives.

Le compte de 1412 vient ainsi démontrer que Jacques I ${ }^{\text {er }}$ et Janus ont su s'adapter aux nouvelles conditions économiques en renforçant l'exploitation directe du domaine de la couronne, en fiscalisant les fiefs, en multipliant les recettes extraordinaires, c'est-à-dire en recourant à des mesures largement répandues dans l'Occident féodal. En 1412, les revenus de la couronne chypriote ne paraissent en rien inférieurs à ceux des États européens de taille comparable et la convoitise des Italiens ne s'exerce pas au hasard. Si la prospérité du premier XIV ${ }^{e} s$. paraît révolue pour Chypre, elle l'est pour nombre d'autres pays. À la mesure du XVe s., le déclin de l'île reste relatif, patent seulement en ce qui concerne le rayonnement de son commerce; dans les années 1410, le royaume connaît la phase de redressement observée en divers ports de la Méditerranée, comme Gênes ou Caffa ${ }^{71}$. Par ailleurs, en laissant de côté les aspects financiers et économiques, qui prétendrait encore résumer par le seul terme de décadence la première partie du règne de Janus, quand le spectacle de la cour émerveillait les princes de l'Italie renaissante?

70 F. BESTA (éd.), op. cit (supra, n. 65) I/I, p. 590.

71 J. DAY, op. cit. (supra, n. 30), I, p. XXXV; M. BALARD, op. cit. (supra, n. 30), II, p. 67 ; id., "Notes sur la fiscalité génoise à Caffa au $x v^{e}$ siècle $n$, Bulletin de la société des antiquaires de France (1993), p. 227-230, 235. 
Appendice

\title{
Civico Museo Correr, cod. Cicogna 3596, pièce ${ }^{\circ} 30$
}

\author{
DELLE COSE DI CIPRO
}

(f. 1 r.)

El stato seu intrada del sublime et potentissimo Re Giano Lusignan Re di Gierusalem, Cipro et Armenia di questo anno $1412^{72}$. Summaria.

Per defati de servitii ${ }^{73}$

Per acordio de servitiii ${ }^{74}$

Per acordio de francomati maridate con pariche $e^{75}$

Per ordinamento del Re posto sopra li feudi de cavallari et altri battendo la decima real ${ }^{76}$

Per feudi de cavallari, et altri che sono in la real ${ }^{77}$

Per feudi pervenuti in la real per 5 anni $^{78}$ b. $134104 / 4$

b. $\quad 68 / 2$

b. $\quad 10 / 16$

b. $10650 / 18$

b. $37331 / 1$

b. $\quad 405 / 21$
72 Dans le royaume de Chypre, comme à Venise, le style en usage pour la datation est celui du $1^{\text {er }}$ mars; l'année fiscale 1412 s'étend donc du $1^{\text {er }}$ mars 1412 au 28 février 1413: J. RICHARD, Documents chypriotes, p. 20-21; J. RICHARD, Le Livre des Remembrances, $\mathrm{p}$. XXX.

$73 \mathrm{Au}$ milieu du $X V \mathrm{e}^{e}$ s., Florio Bustron rappelle les conditions de rachat du servise de cors: "quando il feudato non è d'età, o che il feudo sia pervenuto in femina non maritata, paga de fatto, cioè mancamento di servitio, ducati.... all'anno al signon: F. BUSTRON, p. 462. Le montant des défauts paraît constant entre le milieu du $x v^{e} s$. et le milieu du $X V l^{e}$ s.: en 1436, pour un fief de chevalier, le défaut de service se monte à 800 besants: J. RICHARD, Documents chypriotes, p. 141 ; vers 1550 , il s'élève à 80 ducats pour un service de chevalier, 60 pour un service d'écuyer, 40 pour un service d'hommes d'armes, 10 pour un service de turcople (à cette époque la parité des monnaies est établie à 1 ducat $=10$ besants): F. BUSTRON, p. 462-463; voir éga lement: P. W. EDBURY, art. cit. (supra, n. 31), p. 337-338; J. RICHARD, "Ol $\theta \varepsilon \sigma \mu o i$ ”, p. 342-343.

74 S'agit-il d'un droit de mutation/relief payé au roi par l'héritier d'un fief? Cela semble bien improbable; d'une part, la modestie du droit encaissé en 1412 laisse supposer que l'accord concerne un seul et unique service de corps; d'autre part, le relief n'est mentionné ni par les légistes de l'Orient latin ni dans les actes de la pratique: J. RILEY-SMITH, The Feudal Nobility and the Kingdom of Jerusalem (1973), p. 38,247 n. 116. Peut-être s'agit-il d'un droit particulier perçu à l'occasion d'une nouvelle inféodation.

75 La mobilité de la main-d'œuvre serve du domaine royal a toujours été sévèrement contrôlée; ainsi, une ordonnance du 26 novembre 1297 réitérait l'interdiction d'union d'une parèque à un homme relevant d'un autre statut car ce type de mariage, à défaut d'affranchir la serve, accordait aux enfants la condition du père: RHC-Lois, II, Bans et ordonnances, p. 360 , chap. VII; G. GRIVAUD, "Ordine della secreta di Cipro", p. 592. Notons que dans le compte de 1412 ne figure aucune recette au titre des affranchissements; en 1468, le montant du rachat de la liberté personnelle oscille entre 325 besants (pour une serve) et 375 besants (pour un garçon serf âgé de 15 ans): J. RICHARD, Le Livre des Remembrances, $n^{\circ} 176$.

76 Distinct de la dîme royale, ce poste désigne-t-il une taxe perçue sur les fiefs formés d'assignations établies sur les revenus de la dîme royale? Tel pourrait bien être le cas dans la mesure où des extraits du Livre des Remembrances de l'année 1440 montrent que certains fiefs comprennent des assignations, en nature et en espèces, prélevées sur les revenus de la dîme royale de divers casaux; aucun exemple n'a cependant pu être trouvé pour assurer que des fiefs de chevalier seraient exclusivement composés de ce type d'assignations: ASV, Capi del Consiglio dei Dieci, Dispacci di rettori e altre cariche, b. 290, c. 72 .

77 Les fiefs retournés à la regale depuis plus de cinq ans cf. la ligne suivante - étaient définitivement rattachés aux bailliages royaux (cf. l'exemple mentionné en 1469: J. RICHARD, Le Livre des Remembrances, $n^{\circ} 129$ ); faut-il comprendre qu'ils formaient une réserve dans laquelle le roi puisait pour accorder de nouveaux fiefs?

78 Cette durée de cinq ans ne s'explique guère à travers la littérature juridique; elle peut correspondre à un délai de latence accordé pour épuiser les procédures engagées par des parents vivant hors du royaume, cf. les exemples tirés des fragments des Livres des Remembrances des années 1440-1441: ASV, Capi del consiglio dei Dieci, Dispacci di rettori e altre cariche, b. 290, c. 72 . Au XIII $\mathrm{s}$. dans le royaume de Jérusalem, l'usage laissait un délai d'un an et un jour pour qu'un héritier obtienne une succession: Le livre au roi (éd. M. GREILSAMMER) (1995), chap. 30, p. 218-222; RHC-Lois, I, Livre de Philippe de Novare, chap. LXXXVI, p. 559; RHCLLis, I, Livre de Jean d'Ibelin, chap. CLXXXIV, p. 287 ; RHCLois, II, Abrégé du Livre des Assises des bourgeois, chap. XXXIV, p. 346. 
Per cambii de terreni et altro ${ }^{79}$

$\begin{array}{lc}\text { b. } & 77458 / 20 \\ \text { b. } & 8911 / 9 \\ \text { b. } & 1090 / 8 \\ \text { b. } & 76801 / 9 \\ \text { b. } & 2516 / 12 \\ \text { b. } & 78749 / 14 \\ \text { b. } & 59929 / 9 \\ \text { b. } & 3078 / 15 \\ \text { b. } & 6378 / 21 \\ \text { b. } & 831 /-- \\ \text { b. } & 148 /-- \\ \text { b. } & 4558 /-- \\ \text { b. } & 4400 /-.\end{array}$

79 Cette recette se compose sans doute des bénéfices réalisés au cours des échanges de terres et non de taxes perçues sur l'enregistrement de ces échanges; l'existence de telles taxes ne figure pas dans les actes de la pratique: J. RICHARD, Le Livre des Remembrances, $n^{\text {os }} 60,145$.

80 D'après l'accord conclu entre la Commune de Gênes et Jacques $\left.\right|^{\text {er }}$, le 19 février 1383, les Génois pouvaient continuer d'exploiter des biens à Chypre mais on ne sait à quelles assignations il est ici fait référence, peutêtre à celles levées sur les teintureries royales affermées à des Génois en 1448: L. DE MAS-LATRIE, "Nouvelles preuves de l'histoire de Chypre sous le règne des princes de la maison de Lusignan ", Bibliothèque de l'École des Chartes XXXV (1874), p. 57 ; id., Histoire III, p. 177-178; sur les clauses du traité de 1383: C. OTTEN-FrouX, art. cit. (supra, n. 4), p. 65.

81 Le Livre des Remembrances de l'année fiscale 1468 mentionne plusieurs fermes établies à Nicosie: la boucherie, la fonde (marché) du blé, l'usage des mesures sur cette fonde, les teintureries royales: J. RICHARD, Le Livre des Remembrances, $n^{\text {os }} 77,141,191,193$. En 1367, est aussi mentionnée une fonde du vin: J. RICHARD, "Guy d'lbelin, O.P., évêque de Limassol et l'inventaire de ses biens ", $\mathrm{BCH}$ 74 (1950), p. 123, repris dans id., Les relations entre l'Occident et l'Orient au Moyen Âge (1977), étude $n^{\circ} \mathrm{V}$; on peut également supposer que les moulins de Nicosie, propriétés de la couronne, étaient affermés: J. RICHARD, Documents chypriotes, p. 104. Sur d'autres apauts de Nicosie, voir plus bas, n. 94.

82 La ruga coverta était une rue qui traversait Nicosie d'Ouest en Est; elle longeait le Pédiaios, du château des Lusignan à la place du bas (vers l'actuelle porte de Famagouste); de part et d'autre de cette rue étaient installées des boutiques protégées par de larges auvents, propriétés du roi: F. BUSTRON, p. 463-464.

83 C'est-à-dire les fiés arrestés, fiefs saisis par le roi et dont les revenus sont versés à la Secrète, cf. les exemples publiés par J. RICHARD, Documents chypriotes, p. 146 ; J. RICHARD, Le Livre des Remembrances, nos 166 n. 7, 179.

$84 \mathrm{Au}$ milieu du $X V l^{\mathrm{e}} \mathrm{s}$., les taxes levées à Cérines sont regroupées en une gabelle qui comprend: “... la gabelle exometria, la mesa della porta del mare, capisordi, beccarie, gnafio, meno pulii et altrin. Ces taxes ne peuvent être parfajtement identifiées: une gabelle de la porte terrestre (?), le droit perçu au passage de la porte maritime, des surtaxes (?), le droit sur les boucheries, un droit sur la laine de première qualité, un droit de nettoyage (?); ces droits, qui ne sont pas documentés sous les Lusignan, prècèdent l'installation des Vénitiens à Chypre et devaient, en conséquence, être affermés: Correr, cod. Donà delle Rose 45, f. 203 r. Notons que le terme de misa renvoie à un impôt levé par Hugues IV: F. BALDUCCI PEgOLOTTI, op. cit. (supra, n. 45), p. 85-86; J. RICHARD, "Ol $\theta \varepsilon \sigma \mu o i ́$ ", p. 351.

$85 \mathrm{Au}$ milieu du XIVe s., sont compris dans la gabelle de Pafos: "... l'appalto della praga et... metri, l'appalto delli cuori, del picco, delle pelle conze, della becaria, delle sede, et delle pastellen; on peut y reconnaître: la ferme des pontons et (?), la ferme des cuirs, de la mesure des tissus (pic), des peaux tannées, de la boucherie, des soies et du pastel: Correr, cod. Donà delle Rose 45, f. 203 v.

86 Le même document rapporte que sont compris dans la gabelle de Limassol: "... datio del cerame, del vin, del pan, della carne, del bestiame, delle carobe, delle canute, et tutti altri datii soliti de scodersi dal scrivan della real a Limisso" (le droit sur la cire, sur le vin, sur le pain, sur la viande, sur le bétail, sur les caroubes, les tavernes/canutes, et tous les autres droits habituels versés à l'écrivain de la regale à Limassol): Correr, cod. Donà delle Rose 45, f. 203 v. Le relevé des dîmes levées sur les fermes de la regale à Limassol en 1367 confirment en partie cette liste; sont cités les apauts du comerc (marché), du bain, du criage, du change, de la tannerie, de la mesure des caroubes, du cuir cru: J. RICHARD, Documents chypriotes, p. 78.

87 Dans le bailliage du Carpasse, deux offices étaient affermés à l'époque vénitienne: celui du maréchaucier (chargé du contrôle des droits sur le bétail) et celui du mahtasep (chargé du contrôle des prix, poids et mesures): ASV, Avoga ria di Comun, C. 179 , fasc. 1.

88 Taxe complémentaire levée lors du pesage des marchandises: G. GRIVAUD, "Sur le comerc chypriote de l'époque latine ", in A. A. M. BRYER, G. S. GEORGHALLIDES (éds), The Sweet Land of Cyprus (1993), p. 142.

89 Korikos, sur la côte de la Cilicie arménienne, capturée

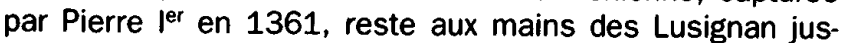
qu'en 1448; les taxes qui y sont perçues sont, sans doute, les douanes auxquelles se réfère MACHAIRAS, $\S 112,567$ (et les commentaires de R. M. DAWKINS, II, p. 96-98). 


\author{
Dala corte del viscontado de Nicosia ${ }^{90}$ \\ Per l'officio del armiraglio ${ }^{91}$ \\ Per intrada di casali non appaltati \\ Per utile tratti dali vini di casali della real
}

(f. 1v)

Per Taette
Per Lassi ${ }^{93}$
Per appalti delli datii de Nicosia ${ }^{94}$
Per appalto dela balanza del fil
Per appalto de Gabelle
Per appalto de terzi de sansarazi
Per altri appalti de Cerines
Per altri appalti de Bafo
Per il baliazo de Carpasso
Per il baliazo de Limisso
Per tanza de dame vidue et vescovi
b. $\quad 3682 /--$
b. $\quad 600 /$ -
b. $249163 / 23$
b. $17250 /$ -

b. $778118 / 10$
90 À défaut des droits d'enregistrement des actes de cession de propriétés bourgeoises, qui restaient normalement à la caisse de la cour du vicomte, il faut voir en ces revenus des amendes imposées sur les jeux de hasard, sur diverses malversations commerciales ou sur l'absence d'hygiène dans les lieux publics: RHC-Lois II, Bans et ordonnances, p. 359 , chap. IV, p. 365 , chap. XV, p. 366 , chap. XVII, p. 372-373, chap. XXIX.

91 L'office de l'amirauté, affermé, était chargé d'établir les rôles des équipages de la marine royale; de 1407 au 2 juin 1414 , Jean de Brunswick, frère de la reine mère, est amiral de Chypre: W. H. RUDT DE COLLENBERG, art. cit. (supra, n. 3), p. 135 n. 302.

92 L'échoite, ou zaette/zaeta en vénitien, correspond à la mainmorte de l'Occident féodal; ce droit levé sur les biens des parèques, à leur décès, est mentionné depuis le XIII' $\mathrm{s}$. Cf. les références citées dans notre étude "Ordine della secreta di Cipro n, p. 584-585.

93 Legs faits par testament, cf. G. BOERIO, Dizionario del dialetto veneziano (1856), p. 362.

94 Ce poste et les trois suivants méritent d'être associés aux fermes de Nicosie mentionnées plus haut (n. 81); ce que comprennent les datii de Nicosie nous est révélé par une notice du milieu du XVI ${ }^{e} s$., qui distingue des droits perçus sur: le pesage du poisson; l'huile, le savon et la fonde du vin; la boucherie; sur les tavernes/canutes situées à l'extérieur de la ville; la teinturerie, la gabelle des camelots, samits, camocas; le pain et la porte; les cuirs crus; la fonde des fruits; les marchandises de la rivière; le suif et les cierges; le poisson frais; le protocuperato dell'aere de Nicosie: Correr, cod. Donà delle Rose 45, f. 203 r-204 r.

95 Ferme du pesage du filé, c'est-à-dire du coton filé destiné aux ateliers de tissage de la ville ou à l'exportation: J. RICHARD, Le Livre des Remembrances, $n^{\circ} 189$.

96 Taxe sur la circulation des marchandises, instituée par
Jacques $\left.\right|^{e r}$, vers 1388-1389; la gabelle était levée aux portes de la ville sur toutes les marchandises entrant à Nicosie ou en sortant: Correr, cod. Donà delle Rose 45, f. 169 r. 97 Correr, cod. Donà delle Rose 45, f. 204 r précise que la ferme du tiers du courtage porte sur les camelots. Cette taxe est déjà mentionnée, dans les années 1330, par F. BALDUCCI PEGOLOTTI, op. cit. (supra, n. 45), p. 87-88.

98 Autres fermes distinguées du poste précédent (cf. supra, n. 84), sans raison apparente.

99 Autres fermes distinguées du poste précédent (cf. supra, n. 85), sans raison apparente.

100 II s'agit, probablement, du produit de la vente de l'office du bailli du Carpasse; cet office est attesté depuis 1298: L. DE MAS-LATRIE, art. cit., (supra, n. 80), p. 50 ; J. RICHARD, Documents chypriotes, p. 65.

101 Attesté depuis 1355: RHCLois II, Bans et ordonnances, p. 377 ; J. RICHARD, Documents chypriotes, p. 65-66.

102 Aucune taxe n'est levée sur les dames nobles qui entrent en veuvage, étant entendu que les veuves âgées de 60 ans ne pouvaient être contraintes de se remarier: $R H C$ Lois I, Livre de Jean d'lbelin, chap. CCXXVIII, p. 362-364; RHC-Lois I, Livre de Philippe de Novare, chap. LXXXVI, p. 559 ; J. A. BRUNDAGE, "Marriage Law in the Latin Kingdom of Jerusalem $n$, in Outremer, Studies presented to J. Prawer (1982), p. 270-271, repris dans id., The Crusades, Holy War and Canon Law (1991), étude $n^{\circ}$ XVII. Sachant qu'une taxe nommée viduazo était prélevée sur les parèques d'un âge inférieur à 60 ans et dont le montant était fixé à 4 besants en 1554, on suppose que la taxe de 1412 concerne les veuves grecques des villes, cf. notre étude "Ordine della secreta di Cipro", p. 580, n. 279. Quant à la taxe sur les évêques grecs, nulle part documentée, elle ne semble pas correspondre à un droit levé lors des investitures, ignoré des textes canoniques: J. DARROUZĖS, "Textes synodaux chypriotes ", REByz 37 (1979), p. 14-37, 55-66. 
Per decima de assegnamenti imposti

\author{
al defato de compagni ${ }^{103}$ \\ Per più giese ${ }^{104}$ \\ Per decimo real ${ }^{105}$ \\ Per vendita de più robe della corte \\ Per follari et caneve del Re \\ Per intrade vecchie
}
b. $\quad 450 /$ -
b. $\quad 600 / .-$
b. $246241 /-$
b. $9600 /$.
b. $4000 /-$
b. $30000 /-$ -

b. $438408 /-.106$

b. $778118 / 14$

summa

b. $1216526 / 14^{107}$

(f. 2r)

oltra il sal delle saline et oltra Famagosta et due leghe che era di Genovesi

Spesa della corte del Re

Di Madama sua madre

Di Madama sua consorte

De li signori soi fratelli et sorelle

Per la maesta del Re

Per formento moza 171 a rason di b. $2 / 12$ il mozo ${ }^{108}$

b. $\quad 427 / 191 / 2$

Per orzo moza $7510 / 11 / 2$ a b. 1 il mozo

b. $\quad 7510 / 41 / 2$

Per vin some 114/7 1/2 a b. 5 la soma

b. $\quad 709 / 14^{109}$

Per cera cantera 4 a b. $3 / 12$ il cantaro ${ }^{110}$

b. $1400 /$ -

Per contadi

b. $4707 / 10$

b. $14755 / 1 / 2^{111}$

Per la measta dela Regina consorte del $\operatorname{Re}^{112}$

Per formento moza $171 /$ - a b. $2 / 12$

b. $\quad 427 / 19^{113}$

103 Libelle assez complexe, qui semble indiquer que le rachat du service d'homme d'armes pouvait être satisfait par des assignations sur lesquelles pesait une décime, mais on ne comprend pas la raison pour laquelle ce poste n'est pas intégré à celui de la dîme royale.

104 Des loyers versés par les églises à la regale?

105 En octobre 1411, Janus avait exempté les commanderies de l'Hôpital de la díme royale, ce qui correpond à un montant total de 2818/17 besants, sans compter les nombreuses remises en nature: L. DE MAS-LATRIE, Histoire II, p. 498-501.

106 Total exact: 338408 besants.

107 Total exact: 1116526 besants et 14 caroubles.

108 Total exact: 427 besants et 12 caroubles.

109 Une erreur s'est introduite dans le texte par le fait d'une résolution erronée de l'unité de mesure; d'une part, la some (saumée) n'est pas une mesure en usage à Chypre au XVes., car l'unité employée est la mètre subdivisée en 8 quarterons, dont chacun valait quatre quartes: J. RICHARD, Documents chypriotes, p. 19. D'autre part, on imagine mal le roi consommant du vin de qualité inférieure (5 besants la saumée $=1$ ou $11 / 4$ besant la mètre) à celle de la troupe de Famagouste en 1468 (prix fixé à 2 besants la mètre), ceci dans une économie où les prix du vin restent stables sur la longue durée: J. RICHARD, Le Livre des Remembrances, $n^{\circ} 64$. Nous proposons donc de corriger: Per vin metri 141/7 1/2 a b. 5 la metre = b. 709/12 1/2. 110 À corriger en : b. 3/12 il rotolo (100 rotoli = 1 cantare $=226,4 \mathrm{~kg})$.

111 Total exact: 14754 besants et 15 caroubles.

112 Charlotte de Bourbon, née vers 1386/1390, avait épousé Janus le 25 août 1411, à Nicosie. De leur union sont issus quatre enfants, dont l'aîné, Jean, naît en 1418: W. H. RUDT DE COLLENBERG, art. cit. (supra, n. 3), p. 154-155.

113 Total exact: 427 besants et 12 caroubles. 
Per vin bianco some $61 / 5$ a b. 5

Per cera lavorata cantara $88 / 4$ a b. $3 / 1 / 2^{115}$

Per contadi à piu rason b. $\quad 306 / 1^{114}$

b. $\quad 309 / 4$

b. $6023 / 10$

b. $\quad 7066 / 10^{116}$

Per li relevi della maesta del Re con le loro nene ${ }^{117}$

Per formento moza 68/3

b. $\quad 181 / 2^{118}$

Per vin some 62/ - - bianco

b. $\quad 305 / 5^{119}$

Per cera rotoli 15 a b. $21 / 2^{120}$

b. $\quad 53 / 51 / 2$

Per conti

b. $1228 / 16$

b. $\quad 1768 / 4^{121}$

Per la capella del $\operatorname{Re}^{122}$, Per vin

b. $\quad 52 / 12$

Per cera

b. $\quad 154 / 14$

Per oglio ostie et incenso

b. $\quad 75 / 20$

b. $282 / 22$

(f. 2v)

Per la stalla, et formenti di cavalli

b. $1200 / \ldots$

Per conzar li ordegni della cuisina

b. $24 /$ -

Per calzamento del Re

b. $80 /-$

Per li bagni real ${ }^{123}$

b. $300 /--$

Per masinatura de biave in altri molini che della real

b. $200 /--$ siando il Re fora per li casali ${ }^{124}$

b. $1000 /-$ -

Per gabella della porta per vin et altro ${ }^{125}$

b. $120 /$.

Per comprita di carta per la corte et fora

Per comprita de piu robe per li cani levrini et bracchi, et setini ${ }^{126}$

b. $\quad 80 /--$

Per comprita de inghistere et gotti

114 À corriger en : metri $61 / 5$ a b. $5=$ b. $308 / 3$.

115 À corriger en : rotoli $88 / 4$ a b. 3/12.

116 Total exact: 7068 besants et 3 caroubles.

117 N'ayant eu aucun enfant de son mariage avec sa première femme, Anglesia Visconti, les enfants du roi dont il est question, en 1412, sont trois enfants naturels: Aloysius, né en 1408, Guy, né vers 1406-1408, et une fille, née avant 1410: id., art. cit., p. 181-184. Sur Guy-Jotin de Lusignan, voir J. RICHARD, "Des Lusignan mythiques au mythe des Lusignan: un "petit Lusignan" au XVe sieccle "in C. MUTAFIAN (éd.), op. cit. (supra, n. 1), p. 251-259.

118 Total exact: 170 besants et $221 / 2$ caroubles.

119 Total exact: 310 besants pour 62 mètres de vin blanc.

120 À corriger en: Per cera rotoli 15 a $3 / 12=$ b. 52/12.

121 Total exact: 1762 besants et $21 / 2$ caroubles.

122 Les dépenses enregistrées ne concernent que les fourni- tures de la chapelle, les salaires des chanteurs devant être assurés par des assignations sur les recettes de la gabelle de la porte de Nicosie, cf. J. RiCHARD, Le Livre des Remembrances, $n^{\circ} 23$. Notons que la vie musicale à la cour de Janus est illustrée par le célèbre manuscrit musical de Turin cf. U. GÜNTHER, L. FINSCHER (éds), op. cit. (supra, n. 58).

123 Ces bains sont visités par Nicolò d'Este: G. GRIVAUD, op. cit. (supra, n. 7), I, p. 46; N. CHRISTOFIDOU, art. cit. (supra, n. 3), p. 175-177.

124 C'est-à-dire lorsque le roi devait, durant ses chevauchées à travers le pays, faire moudre son blé dans les moulins de particuliers.

125 Le roi ne disposait donc pas d'une franchise totale sur l'introduction des vins et d'autres denrées à Nicosie.

126 Poste qui recouvre seulement les dépenses accomplies pour l'entretien du chenil royal; setino: tissu de soie très fin. 
Per comprita di spetie per fare umclare ${ }^{127}$

Per semenze menute ale giostre et alle festa del San Gianni ${ }^{128}$

Per cavalcate del Re, et per quelli che degiunano in corte ${ }^{129}$

Per gratie ch'el Re fa a contori, medici et altri ${ }^{130}$

Per spese de robe in li bagni

Per lavar le tovaglie, et tovaglioli

Per altre spese di cani

Per spese delli huomeni d'arme in la Terra et fuora

Per la panataria, o, spesa del forno ${ }^{131}$

Per legne per le camere dela corte

Per comprita de tovaglioli, et mantili

Per spese di falconi et falconieri ${ }^{132}$

Per salariati del Re in la corte ${ }^{133}$

Per salariati dela Regina consorte del $\operatorname{Re}^{134}$
b. $\quad 100 /--$
b. $\quad 400 /--$
b. $\quad 795 /--$
b. $2000 /$ -
b. $\quad 482 / 12$
b. $60 /$ -
b. $\quad 31 /$ -
b. $9600 / .-$
b. $250 /$ -
b. $300 /-$ -
b. $200 /$ -
b. $\quad 3131 / 12$
b. $24577 / 2$
b. $7430 /$ -

b. $52461 / 2$

Per la maesta della Regina madre der $\mathrm{Re}$, et suoi figli videlicet

$$
\begin{aligned}
& \text { Per la Regina }{ }^{135} \\
& \text { Per suo figlio eletto }{ }^{136} \\
& \text { Per il contestabile } \\
& \text { Per madama Isabella } \\
& \text { Per il Sijnescalco } \\
& \text { Per il Principe }
\end{aligned}
$$

$\begin{array}{ll}\text { b. } & 5471 / 21 \\ \text { b. } & 2505 / 1 / 2 \\ \text { b. } & 2572 / 1 / 2 \\ \text { b. } & 2505 / 1 / 2 \\ \text { b. } & 2496 / 231 / 2 \\ \text { b. } & 2496 / 231 / 2\end{array}$

127 Probablement des épices nécessaires à la fabrication d'onguents pour les inhumations.

128 Ainsi, des joutes sont organisées à l'occasion de la visite de Nicolò d'Este: G. Grivaud, op. cit (supra, n. 7), I, p. 43.

129 Notamment pour les pèlerins qui se rendent à Nicosie, au retour du voyage de Jérusalem, cf. le récit des fastueuses réceptions organisées en l'honneur du marquis Nicolò d'Este: ibid., p. 41-49.

130 Exemples de largesses à un jongleur turc en juin 1412: ibid., p. 47.

131 Autres mentions de la paneterie de l'hôtel du roi, en 1468 : J. RICHARD, Le Livre des Remembrances., nos 91 , 140 , p. XIX.

132 En 1468, deux fauconniers sont attachés à l'hôtel du roi: J. RICHARD, Le Livre des Remembrances., $n^{\text {os }} 53,74$, $82,90$.

133 Le personnel salarié de la cour comprend, outre les serviteurs du palais, les officiers et secrétains de la Secrète; certains d'entre eux percevaient également une partie de leur salaire sous forme d'assignations, mais rien n'assure que le montant de ces assignations soit compris dans ce poste.
134 Poste qui concerne sans doute le personnel français qui avait suivi Charlotte de Bourbon à Nicosie, cf. MACHAIRAS, $\S 642$.

135 Helvis de Brunswick avait épousé Jacques $\left.\right|^{\text {er }}$, le $1^{\text {er juin }}$ 1365, et en avait eu au moins treize enfants; elle meurt à Nicosie, le 15 janvier 1422: W. H. RUDT DE COLLENBERG, art. cit. (supra, n. 3), p. 134-135.

136 Hugues de Lusignan, né vers 1391, cardinal en 1426 , mort à Genève en août 1442, avait reçu la commende de l'archevéché de Nicosie vers 1411: id., "Les cardinaux de Chypre Hugues et Lancelot de Lusignan ", Archivum historiæ pontificiæ 20 (1982), p. 88-115, 88-91 plus spécialement. 137 Philippe de Lusignan, né entre 1376 et 1385 , attesté connétable de Chypre de 1401 à 1414 , mort vers 1428 1432 : id., art. cit. (supra, n. 3), p. 155-156.

138 Isabelle de Lusignan, née avant 1382, morte avant 1432: ibid., p. 166-167.

139 Eudes de Lusignan, né vers 1385-1390, sénéchal de Jérusalem, quitte Chypre vers 1420 pour se placer au service d'Alphonse $V$ d'Aragon; il meurt en février 1421, à Palerme: ibid, p. 159.

140 Henri de Lusignan, né vers $1380-1385$, reçoit le titre de Prince de Galilée entre 1406 et 1412 , meurt lors de la bataille de Choirokitia ( 7 juillet 1426): ibid., p. 156-158. 
Per madama Agnese ${ }^{141}$

Per assegnamenti che paga a feudati et stipendiati

somma
Lintrada del Regno
La spesa tutta
\[ \begin{array}{l}\text { b. } 1216526 / 14 \\ \text { b. } 337352 / 10\end{array} \]
$\begin{aligned} & \text { oltre le saline } \\ & \text { et li mancava ancora Famagosta con do leghe } \\ & \text { la qual era in mano de Genovesi. }\end{aligned}$

b. $2489 / 1$

b. $240481 / 21$

b. $261018 / 191 / 2$

$52461 / 2$

$282 / 22$

$1768 / 4$

$7066 / 10$

$14755 / 1 / 2$

b. $337352 / 10^{142}$
141 Agnès de Lusignan, née vers 1382, morte à Venasque, le $1^{\text {er }}$ mars 1459: ibid., p. 167-168; N. CHRISTOFIDOU, "H

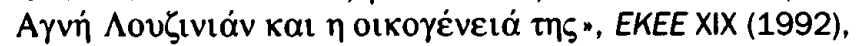
p. $214-250$.
142 Compte tenu des erreurs qui se sont glissées dans les pages précédentes, le total des dépenses s'élève à 337353 besants et $51 / 2$ caroubles.

143 Total exact: 779173 besants et $81 / 2$ caroubles. 